\title{
Effectiveness and safety of beta blockers in the management of hypertension in older adults: a systematic review to help reduce inappropriate prescribing
}

Anna Vögele ${ }^{1 *}$, Tim Johansson ${ }^{2}$, Anna Renom-Guiteras ${ }^{3,4}$, David Reeves ${ }^{5}$, Anja Rieckert $^{3}$, Lisa Schlender ${ }^{3}$, Anne-Lisa Teichmann ${ }^{3}$, Andreas Sönnichsen ${ }^{3}$ and Yolanda V Martinez ${ }^{5}$

\begin{abstract}
Background: The benefit from a blood pressure lowering therapy with beta blockers may not outweigh its risks, especially in older populations. The aim of this study was to look for evidence on risks and benefits of beta blockers in older adults and to use this evidence to develop recommendations for the electronic decision support tool of the PRIMA-eDS project.

Methods: Systematic review of the literature using a stage approach with searches for systematic reviews and meta-analyses first, and individual studies only if the previous searches are inconclusive. The target population were older adults ( $\geq 65$ years old) with hypertension. We included studies reporting on the effectiveness and/or safety of beta blockers on clinically relevant endpoints (e.g. mortality, cardiovascular events, and stroke) in the management of hypertension. The recommendations were developed according to the GRADE methodology.

Results: Fifteen studies were included, comprising one meta-analysis, four randomized controlled trials, six secondary analyses of randomized controlled trials and four observational studies. Seven studies involved only older adults and eight studies reported subgroup analyses by age. With regard to a composite endpoint (death, stroke or myocardial infarction) beta blockers were associated with a higher risk of events then were other antihypertensive agents. Further, beta blockers showed no benefit compared to other antihypertensive agents or placebo regarding mortality. They appear to be less effective than other antihypertensive agents in reducing cardiovascular events. Contradictory results were found regarding the effect of beta blockers on stroke. None of the studies explored the effect on quality of life, hospitalisation, functional impairment/status, safety endpoints or renal failure.
\end{abstract}

Conclusion: The quality of current evidence to interpret the benefits of beta blockers in hypertension is rather weak. It cannot be recommended to use beta blockers in older adults as first line agent for hypertension.

Keywords: Systematic review, Beta blockers, Aged, Hypertension, Effectiveness, Inappropriate prescribing

\footnotetext{
* Correspondence: anna.voegele@sakam.it

${ }^{1}$ South Tyrolean Academy of General Practice, via dei Vanga, Bolzano, Italy

Full list of author information is available at the end of the article
} 


\section{Background}

Hypertension is an important public health challenge worldwide as nearly 1 billion people or $\sim 26 \%$ of the adult population of the world suffers from hypertension $[1,2]$. In Europe, the overall prevalence of hypertension appears to be around $30-45 \%$ of the general population [3], with a steep increase with ageing [4-6]. Hypertension is a major risk factor for cardiovascular and renal disease, and numerous clinical trials including studies in older adults have documented that effective treatment improves survival and confers cardiovascular benefits [4, 5]. The Framingham Heart Study and a meta-analysis done by Lewington et al. have shown a continuous and positive relationship between cardiovascular events and usual blood pressure above a baseline level of approximately $115 / 75 \mathrm{mmHg}$ at all ages and in both sexes [6, 7]. Although the strength of the association is weakened with age [7], the absolute difference in cardiovascular risk between the highest and lowest usual blood pressure levels is much greater in older subjects [8]. Hence, it could be expected that especially older adults benefit from a blood pressure lowering therapy. However, there is a phenomenon called "reverse epidemiology" in older adults suffering from hypertension [9] which describes that higher blood pressure can be associated with increased survival [10]. The phenomenon likely reflects the confounding effects of other comorbid conditions that are quite common in this age group. This means that a lower blood pressure may be a proxy for poor health and a warning sign that further blood pressure lowering may actually be harmful. Thus, lower - for older adults - may not always be better [8]. Moreover, some studies suggest that in older adults tight blood pressure control (systolic blood pressure $<140 \mathrm{mmHg}$ ) may not reduce stroke more efficiently than a goal blood pressure $<150 / 90$. In adults aged $\geq 75$ years tight blood pressure may increase the incidence of cardiac and vascular disease, and renal failure [11, 12]. Tight blood pressure control is not recommended for patients with dementia or cognitive impairment or functional impairment and in patients with limited life expectancy $(<2$ years $)$ [13] .

Drugs used in treating hypertension are among the most commonly used drug classes that are associated with preventable drug-related hospital admissions especially in older populations $[14,15]$. Among antihypertensive drugs, particularly beta blockers appear to be a problematical drug class for the treatment of hypertension [16]. A recently published Cochrane review on beta blockers for hypertension concludes that beta blockers are not recommended as first line treatment for hypertension as compared to placebo due to their modest effect on stroke and no significant reduction in mortality or coronary heart disease. Compared to other drug classes (diuretics, calcium channel blockers, and renin angiotensin system inhibitors) beta blockers were not efficient in decreasing mortality and morbidity [17]. It is argued that the use of beta blockers in older adults may not be justified because of physiologic changes in people over 60 years of age. These include a low cardiac output, bradycardia, high total peripheral resistance, reduced renal blood flow and glomerular filtration rate, and low plasma renin activity [18].

Nevertheless, to the best of our knowledge, so far no systematic review has analysed the specific evidence on the use of beta blockers for the management of hypertension in aged populations. This systematic review aims to explore the effectiveness and safety of beta blockers in the treatment of hypertension in older adults ( $\geq 65$ years). A further aim was to develop recommendations on when to discontinue or to adjust the dose when using beta blockers in the treatment of hypertension in older adults. These recommendations were implemented in the electronic decision support tool of the PRIMAeDS project (Polypharmacy in chronic diseases: Reduction of Inappropriate Medication and Adverse drug events in elderly populations by electronic Decision Support, www.prima-eds.eu).

\section{Methods}

This systematic review was developed following an adaptation of the methods proposed by both the Cochrane Handbook for Systematic Reviews of Interventions [19] and the Preferred Reporting Items for Systematic Reviews and Meta-Analyses (PRISMA) [20]. A full description of the methods has been published previously [21]. A protocol for this systematic review has been elaborated and it is available from the authors on request.

\section{Study inclusion criteria \\ Types of studies}

We included systematic reviews, meta-analyses, controlled interventional studies and observational studies reporting on risks and benefits of the use of beta blockers in the treatment of hypertension in older adults.

\section{Types of participants}

Older adults, $\geq 65$ years old, with arterial hypertension were included. We ensured, that there was a sufficient number of older adults using the following criteria by type of design.

For systematic reviews and meta-analyses (any of the following criteria):

- overall mean or median age $\geq 65$ years,

- overall mean or median age $<65$, but subgroup analysis of participants $<65$, or 
- overall mean or median age not reported but more than $80 \%$ of studies reported a mean or median age $\geq 65$ years.

For controlled interventional studies and observational studies (any of the following criteria):

- $\geq 80 \%$ of participants $\geq 65$ years or

- $<80 \%$ of participants $\geq 65$ years but subgroup analysis of participants $<65$. We included any setting reporting on the management of hypertension using beta blockers.

\section{Types of interventions}

We included studies reporting on the effectiveness and/ or safety of beta blockers for the management of hypertension. Studies were included irrespective of beta blockers prescribed as monotherapy or in combination with any other drug for the treatment of hypertension. We included studies comparing beta blockers versus placebo, no treatment, other antihypertensive drugs or a non-pharmacological intervention.

\section{Types of outcomes}

We included any of the following clinically relevant endpoints as primary outcomes:

- Mortality

- Hospitalization

- Cardiovascular event including stroke

- Quality of life

- Adverse drug event

- Life expectancy

- Cognitive impairment or cognitive status

- Functional impairment or functional status

- Renal failure

- Composite end points including any of the above

- Any of the above evaluated as safety endpoints

\section{Study exclusion criteria}

We excluded conference abstracts, pooled analyses, editorials, opinion papers, case reports, case series, narrative reviews, letters, and qualitative studies. We excluded studies evaluating only blood pressure values.

\section{Search method}

Database searches were conducted by YVM and AW. We performed a stage approach with searches 1 and 2 in various databases for systematic reviews and metaanalyses, and search 3 for individual studies. During study selection for search 1 and 2, we identified eligible individual studies from excluded systematic reviews and meta-analyses and transferred those to search 3A for potential inclusion. The list of studies in search $3 \mathrm{~A}$ was checked for inclusion following the procedures described in the section "Selection of studies". Search 3B was designated as a last step to identify controlled interventional studies and observational trials in various databases. The respectively following search was performed only if the prior searches were inconclusive. We did not apply any language restriction to the search. The following paragraphs contain detailed information about search stages, databases and search dates:

- Search 1 was conducted on 11 September 2013 and updated on 22 December 2015 in the Cochrane Database of Systematic Reviews (OVID interface, 2005 to December 2015) and the Database of Abstracts or Reviews of Effects (DARE, OVID interface, 1991 to 2nd Quarter 2015).

- Search 2 was conducted on 17 October 2013 and updated on 22 December 2015 in MEDLINE (OVID interface, In-Process \& Other Non-Indexed Citations 1946 to November Week 3 2015), EMBASE (OVID interface, 1974 to 2015 December 21), Health Technology Assessment (HTA, OVID interface 2001 to 4th Quarter 2015) and International Pharmaceutical Abstracts (IPA, OVID interface 1970 to December 2015).

- Search 3A consisted of eligible individual studies identified from systematic reviews and meta-analyses excluded from searches 1 and 2 covering the time of these searches.

- Search 3B was conducted on 28 September 2016 in MEDLINE (OVID interface, 2005 to 2016), EMBASE (OVID interface, 2005 to 2016), HTA (OVID interface 2005 to 2016) and IPA (OVID interface 2005 to September 2016). The result of search 3B was limited to the last 5 years (1 January 2011 to 28 September 2016) because search 3A had already used a high quality Cochrane review [17] which had covered all individual studies eligible for the time before 2012 (see 3A). This Cochrane review has recently been updated, and again, no additional eligible studies for our review were identified [22].

- Our hand search comprised a review of the bibliographies of all included studies.

- Study protocols from all searches were also collected to consider for future updates of this systematic review.

The PICOS-framework was used to develop the search strings. A selection of keywords used were: Population (older adults, aged) AND Condition (high blood pressure, hypertension) AND Intervention (beta blocker) AND Outcome (quality of life, mortality, life expectancy, hospitalization, cognitive impairment, functional impairment, cardiovascular event including stroke, renal failure) 
AND Study designs (systematic reviews, meta-analyses, controlled interventional studies and observational studies). Additional file 1 shows the full search strings. Endnote $\mathrm{X} 7$ was used to retrieve search results and to deduplicate references.

\section{Selection of studies and data management}

First, BF, AV and TJ assessed titles and abstracts from search 1 and 2 (including the search update) and identified studies to include using Endnote X7. Search 3A was done by YVM, TJ and AV, and search $3 B$ by CS, AR, ALT, LS and BF. Second, full manuscripts were obtained for all titles and abstracts that appeared to meet the inclusion criteria or for cases of uncertainty for inclusion. ARG, YVM or AS were consulted when the two researchers could not reach an agreement on whether to include a study.

\section{Data extraction}

Two reviewers (AV, YVM or TJ respectively CS, AR, ALT, LS, SW or BF) independently conducted data extraction of the included studies using a standardised and piloted data collection form which was published with the protocol [23]. The two reviewers checked each other's data extraction to look for completeness and accuracy. The data collection form included information related to the study design and aim, characteristics of the participants (i.e. age, sex, setting, comorbidity, use of concomitant medications, frailty, functional and cognitive status), the intervention (i.e. beta blockers), comparison, time to follow-up, and reported outcomes.

\section{Quality appraisal}

We used three validated assessment tools to evaluate the quality of each study design: for systematic reviews/ meta-analyses the measurement tool to assess systematic reviews (AMSTAR) $[24,25]$ for intervention studies the Cochrane Collaboration's tool for assessing risk of bias [26] and for observational studies the Critical Appraisal Skills Programme (CASP) [27, 28]. Risk of bias was assessed independently by at least two review authors according to the Cochrane Handbook [26].

\section{Data synthesis}

Methods utilised to synthesise the studies depended on their quality, design and heterogeneity. According to the protocol we wanted to pool results of studies if at least two were homogeneous regarding participants, interventions and outcomes [23]. If that was not possible due to differences in the reporting or substantive heterogeneity, we reported a narrative synthesis describing all included studies, participants and findings. No additional metaanalyses were performed.

\section{Identification of additional "references of interest" and the development of recommendations}

During the search process, BF, AV, TJ, YVM and ARG identified additional references for the development of recommendations according to the methodology described by Martinez-Renom Guiteras et al. [21]. We identified nine additional references which can be seen in Additional file 2. Two were found in search 2 , one in the search update, one in the hand search of references of included studies, two were suggested by two clinical researchers (IK and MMV) and three were found by snowballing [29]. The studies did not meet our inclusion criteria (mainly due to age $<65$ years), but provided supportive information for the formulation of the recommendations. Included studies and additional references were summarised in a document that was used in team meetings to discuss recommendations on when the use of beta blockers should be discontinued or new doses should be considered in older adults for the management of hypertension [21]. Each recommendation was given strength (weak or strong) and quality (low, moderate or high) following the GRADE methodology [30-32]. The recommendations are provided in Table 1.

\section{Results \\ Results of the search}

We identified 1449 records through database searching and 434 through other sources (hand search of reference lists of included studies). After removing 116 duplicates, we screened 1767 records and excluded 1543 records checking titles and abstracts. We assessed 224 full texts for eligibility and excluded 209 records. Additional file 3 provides the comprehensive list of reasons for exclusion of studies after full text analysis. The PRISMA flow diagram is presented in Fig. 1.

\section{Characteristics of the included studies}

We included one meta-analysis [33], four randomized controlled trials [34-37], six secondary analyses (post hoc analyses) of randomized controlled trials [36, 3842], and four observational studies [43-46] (three cohort studies and one cross sectional study). Table 2 shows details of the included meta-analysis, and Table 3 shows details of the included trials. Follow-up in the included trials ranged from 9 months [36] to a mean of 5.8 years [35]. Six studies were carried out in the UK [35-39, 42], two in Japan [41, 47] and single studies in Italy [46], Sweden [43], and the USA [45]. Two studies were multicentre trials conducted in European countries [40, 44], and one study was conducted in 14 countries worldwide [34]. 
Table 1 Recommendations for beta blockers in older people with hypertension

\begin{tabular}{|c|c|c|c|}
\hline Recommendation & $\begin{array}{l}\text { Strength of the } \\
\text { recommendation }\end{array}$ & Quality of the evidence & Type of evidence \\
\hline $\begin{array}{l}\text { It is suggested to discontinue the beta blocker } \\
\text { or change it to another antihypertensive drug } \\
\text { (unless another indication for beta blockers } \\
\text { exists), because beta blockers may increase } \\
\text { the risk of stroke and other composite } \\
\text { cardiovascular outcomes compared to other } \\
\text { antihypertensive agents while not revealing } \\
\text { any benefit regarding cardiovascular } \\
\text { outcomes or mortality compared to placebo } \\
\text { for adults }>60 \text { years. }\end{array}$ & Strong & $\begin{array}{l}\text { Low } \\
\text { Downgraded for indirectness } \\
\text { because only one meta-analysis } \\
\text { and one RCT were focused on } \\
\text { older people }\end{array}$ & $\begin{array}{l}2 \text { Cochrane reviews }[17,49], 2 \text { meta-analyses } \\
{[33,54], 2 \text { recommendation papers from the }} \\
\text { Canadian Hypertension Education Program } \\
{[51,52] \text {, and } 1 \text { RCT }[35]}\end{array}$ \\
\hline $\begin{array}{l}\text { It is suggested to discontinue atenolol for the } \\
\text { management of hypertension because it } \\
\text { appears to be less effective than other } \\
\text { antihypertensives in reducing cardiovascular } \\
\text { events, and to have a higher risk of adverse } \\
\text { events. }\end{array}$ & Strong & $\begin{array}{l}\text { Low } \\
\text { Downgraded for indirectness } \\
\text { because only one RCT was } \\
\text { focused on older people }\end{array}$ & 2 Cochrane reviews $[17,50]$ and 2 RCTs $[35]$ \\
\hline $\begin{array}{l}\text { It is suggested to discontinue beta blockers as } \\
\text { monotherapy for the management of } \\
\text { hypertension because it may be inferior to } \\
\text { other antihypertensives in preventing stroke, } \\
\text { and not to have any benefits in decreasing } \\
\text { the rates of cardiovascular events. This } \\
\text { recommendation does not apply if the patient } \\
\text { has other indications for beta blockers (heart } \\
\text { failure, arrhythmia, previous myocardial } \\
\text { infarction, angina pectoris). }\end{array}$ & Strong & $\begin{array}{l}\text { Low } \\
\text { Downgraded for indirectness } \\
\text { because only the meta-analysis } \\
\text { included a subgroup analysis in } \\
\text { older people }\end{array}$ & $\begin{array}{l}1 \text { Cochrane review [17], } 1 \text { meta-analysis [33] } \\
\text { and } 1 \text { evidence based guideline [38] }\end{array}$ \\
\hline
\end{tabular}

\section{Participants}

Table 4 shows the characteristics of the participants in the included studies. Nine studies analysed older adults (age $\geq 65$ years) $[35,36,39,42-46,48]$ and another fife studies included a subgroup analysis of patients 65 years or older $[34,37,40,41,47]$. The meta-analysis performed a comparison between studies enrolling patients $<60$ years vs. those enrolling patients $\geq 60$ years in a subgroup analysis [33]. The studies included in this group of studies enrolling patients $\geq 60$ years met our inclusion criteria as nine out of the 11 reported a mean age of participants of $\geq 65$ years ( $82 \%$ of the studies with mean age $\geq 65$ years) [33]. Male sex ranged from 29\% [49] to $100 \%$ [45]. Two trials reported ethnicities [34, 45]. Comorbidity was reported in all studies except in the MRC trial [35]. Six studies reported on use of concomitant medications [34, 41, 43, 44, 46, 47]. Frailty and/or cognitive status were only reported in two studies $[45,46]$.

\section{Interventions and outcomes}

Detailed information about interventions and outcomes can be found in Table 3: Summary of characteristics of the included studies.

\section{Any beta blocker}

The meta-analysis [33] included only randomized controlled trials evaluating the efficacy of beta blockers as first-line therapy for hypertension in preventing major cardiovascular events (i.e. stroke, myocardial infarction or death). In the analyses of the group of studies that we are including, the authors compared beta blockers to no treatment, placebo, diuretics, ACE-inhibitors, calciumchannel blockers and angiotensin-receptor blockers. The Matsuzaki trial [47] and the related secondary analysis of Ogihara et al. [41] analysed the optimal calciumchannel blocker benidipine-based combination therapy comparing three intervention arms, one with additional angiotensin receptor blocker, one with a beta blocker and one with a thiazide diuretic. The primary outcomes of these studies were the composite of cardiovascular morbidity and mortality. The observational study of Carlsson et al. [43] compared the following beta blocker treatments: selective vs. no treatment, non-selective no treatment and non-selective vs selective with regard to the outcome of mortality. Gelber et al. [45], another observational study, analysed beta blocker alone and beta blocker in combination with one other antihypertensive drug for the outcome of the development of cognitive impairment.

\section{Atenolol}

Atenolol (50 or $100 \mathrm{mg} / \mathrm{d}$ ) was examined in three randomized controlled trials [34, 35] and the related secondary analyses [36, 38, 39, 42], a post hoc analyses of a randomized controlled trial [40] and in two observational 


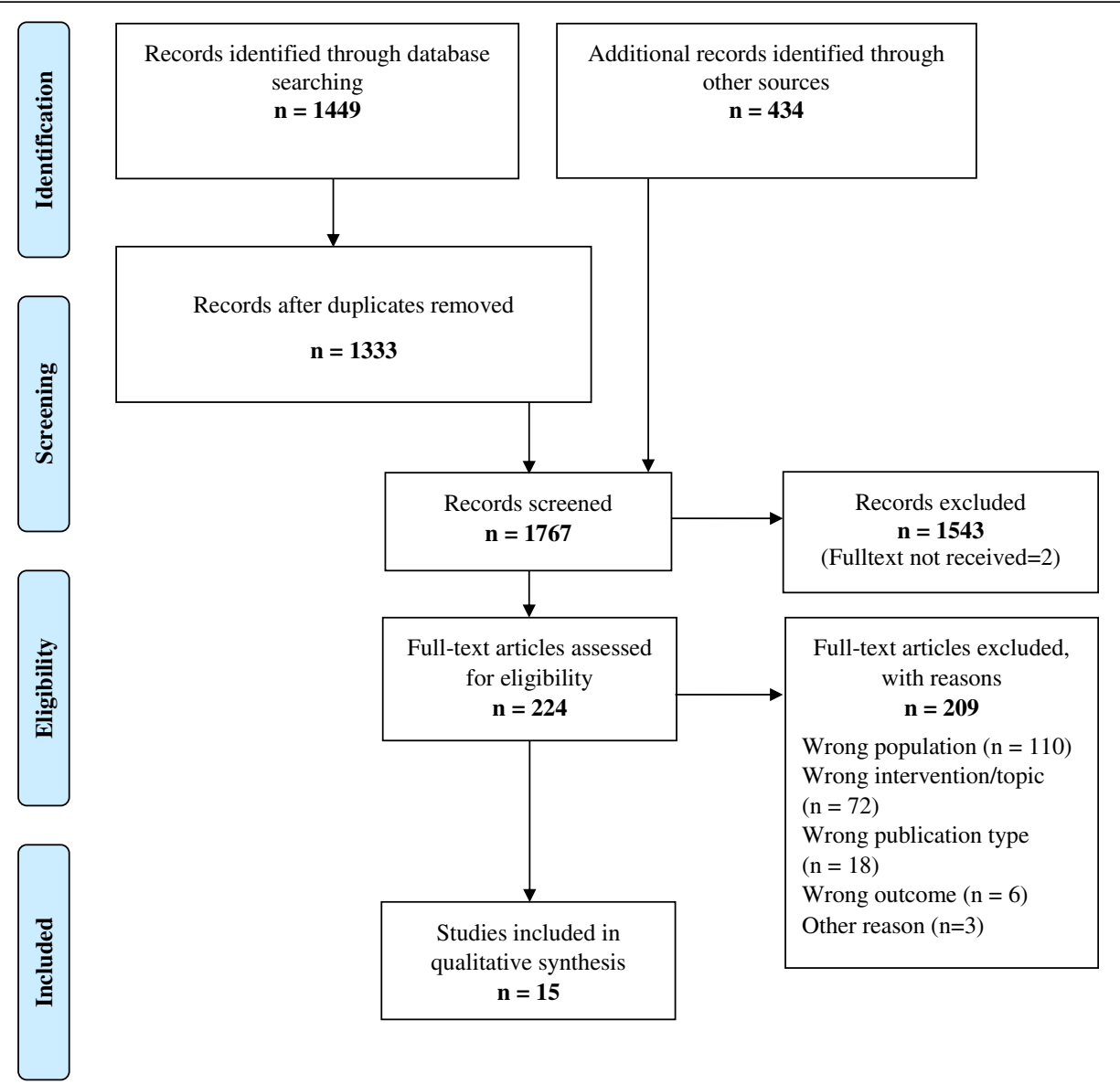

Fig. 1 Preferred Reporting Items for Systematic Reviews and Meta-Analyses (PRISMA) flow diagram

studies [44, 46]. In Pepine et al. [34] atenolol was compared to a treatment with verapamil, in the MRC trial [35] and its related secondary analyses it was compared with hydrochlorothiazide plus amiloride (Moduretic $\odot$ ) or placebo [36, 38, 39, 42], and in Coope et al. [34] atenolol was compared to no treatment. In Ruwald et al. [40], atenolol was compared to losartan plus hydrochlorothiazide and in the observational study of Collier et al. [44], an atenolol based treatment was compared with an amlodipine based treatment. The observational study of Testa et al. [46] compared atenolol to no- atenolol. The primary outcomes of these trials can be seen in Table 3.

\section{Main findings}

The results of the included studies are displayed in Tables 5 and 6 and summarized below.

\section{Composite outcome of death, nonfatal stroke and nonfatal myocardial infarction}

The meta-analysis [33] found that no reduced event rates for the composite outcome were found in beta blocker treatment groups compared to placebo in trials enrolling older adults (relative risk RR 0.89, 95\% confidence interval CI 0.75-1.05). But in the comparison with other antihypertensive agents, a higher risk of events was found in the beta blocker- group (RR 1.06, 95\% CI 1.01-1.10). In the subgroup analysis of one of the included randomized controlled trials [34], no significant difference in the first occurrence of the composite outcome was found between the verapamiltreatment-group and the atenolol-treatment-group (RR 0.93, 95\% CI 0.84-1.03). Ruwald et al. [40] analysed the combined endpoint of cardiovascular death, nonfatal stroke and nonfatal myocardial infarction and found that in patients $\geq 67$ years losartan significantly reduced the risk of this combined endpoint compared to atenolol (hazard ratio HR 0.79, 95\% CI 0.69-0.91).

\section{Mortality}

The analysis of the included group of studies of the meta-analysis [33] found no appreciable impact on rates of death, neither comparing beta blocker treatment- and placebo- groups (RR 0.91, 95\% CI 0.74-1.12), nor comparing beta blocker- and other antihypertensive agentgroups (RR 1.05, 95\% CI 0.99-1.11) The MRC trial [35] confirms this results (RR 1.06, 95\% CI 0.90-1.27, 
Table 2 Data extraction of the meta-analysis

\begin{tabular}{|c|c|}
\hline \multicolumn{2}{|c|}{ Khan et al. 2006. Re-examining the efficacy of $\beta$-blockers for the treatment of hypertension: a meta-analysis, CMAJ, 174(12): 1737-42 [33] } \\
\hline Country & Canada \\
\hline Funding & Not stated \\
\hline Setting & Not stated \\
\hline Objective & $\begin{array}{l}\text { To explore the efficacy (stroke, myocardial infarction and death) of beta blockers in different } \\
\text { age groups }\end{array}$ \\
\hline \multicolumn{2}{|r|}{ Inclusion and exclusion criteria } \\
\hline Population & $\begin{array}{l}\text { Hypertension, distinguish between "younger" patients }<60 \text { years (mean age ranged from } 45.5 \\
\text { to } 56.2 \text { years, } n=10 \text { trials and } n=50,612 \text { patients) and "older" patients } \geq 60 \text { years ( } 60.4 \text { to } 76 \\
\text { years, } n=11 \text { trials and } n=95,199 \text { patients, } 9 \text { trials out of } 11 \text { with mean age } \geq 65 \text { years). }\end{array}$ \\
\hline Intervention & Beta blocker as first-line therapy for hypertension in preventing major cardiovascular events \\
\hline Control & $\begin{array}{l}\text { No treatment, placebo, diuretic, ACE inhibitor, calcium-channel blocker, angiotensin-receptor } \\
\text { blocker }\end{array}$ \\
\hline Outcomes & Stroke, myocardial infarction, or death \\
\hline Study designs & Randomized controlled trials \\
\hline
\end{tabular}

\section{Methods}

Study design

Systematic review including meta-analysis. Results of the individual studies are combined to produce an overall statistic.

Last date searched

18 January 2006

Data bases searched

PubMed (1950-18.01.2006)

Other sources searched

Hand search, reference lists of published hypertension meta-analysis (MEDLINE) and the Cochrane Library. Contacted Canadian hypertension experts.

Number of included studies

21 Randomized controlled trials

Number of included patients

145,811

\section{Outcomes, results}

\section{Primary}

Composite cardiovascular outcome of death, nonfatal myocardial infarction or nonfatal stroke

Beta blockers reduced event rates compared with placebo (RR 0.86, 95\% Cl 0.74-0.99, based on 794 events in 19,414 patients), in trials enrolling younger patients, but benefits were not found in trials enrolling older patients (RR $0.89,95 \%$ Cl $0.75-1.05$, based on 1115 events in 8,019 patients).

Among 30,412 patients younger than 60 years of age, there was no difference in event rates between those randomly assigned to beta blockers therapy compared with those receiving other antihypertensive agents ( 1515 events, RR $0.97,95 \% \mathrm{Cl} 0.88-1.07)$. However, in the 79,775 patients 60 years of age or older, beta blockers were associated with a higher risk of events than other antihypertensive agents (7405 events, RR 1.06, 95\% Cl 1.01-1.10).

\section{Secondary}

\section{Beta blocker compared to placebo}

\section{"Younger}

population" $<60$

years

Death

RR $0.94,95 \%$ Cl

$0.79-1.10$

Nonfatal myocardial infarction

RR $0.85,95 \% \mathrm{Cl}$

$0.71-1.03$

Nonfatal stroke

RR $0.84,95 \% \mathrm{Cl}$ $0.65-1.10$

Heart failure

RR $1.05,95 \% \mathrm{Cl}$

0.72-1.54

Beta blocker compared to other antihypertensive agents

\section{"Younger} population" $<60$

years

Death

RR $0.97,95 \%$ C $0.83-1.14$

Nonfatal myocardial infarction

RR $0.97,95 \%$ Cl RR $1.06,95 \%$ Cl $0.94-1.20$
“Older population" $\geq 60$ years

RR $0.91,95 \%$ Cl 0.74-1.12

RR $0.98,95 \%$ Cl 0.83-1.16

RR $0.78,95 \%$ Cl 0.63-0.98

RR $0.54,95 \%$ Cl $0.37-0.81$

"Older population" $\geq 60$ years

RR $1.05,95 \%$ Cl 0.99-1.11 
Table 2 Data extraction of the meta-analysis (Continued)

\begin{tabular}{|c|c|c|}
\hline Nonfatal stroke & $\begin{array}{l}\text { RR } 0.99,95 \% \mathrm{Cl} \\
0.67-1.44\end{array}$ & RR 1.18, 95\% Cl 1.07-1.30 \\
\hline \multirow[t]{2}{*}{ Heart failure } & $\begin{array}{l}\text { RR } 0.93,95 \% \mathrm{Cl} \\
0.64-1.34\end{array}$ & RR $0.98,95 \%$ Cl 0.87-1.11 \\
\hline & \multicolumn{2}{|c|}{ Subgroup analysis, $\geq 60$ years } \\
\hline \multicolumn{3}{|c|}{$\begin{array}{l}\text { Beta blocker compared to placebo or no treatment } \\
\text { ( } 5 \text { trials and } n=8,019 \text { patients, range mean age } 65 \text { to } 75.7 \text { years) }\end{array}$} \\
\hline $\begin{array}{l}\text { Composite cardiovascular outcome of death, } \\
\text { nonfatal myocardial infarction or nonfatal stroke }\end{array}$ & \multicolumn{2}{|c|}{$\begin{array}{l}\text { Beta-blockers' benefits were not found in trials enrolling older patients } \\
\text { RR } 0.89,95 \% \mathrm{Cl} 0.75-1.05 \text {, based on } 1,115 \text { events in 8,019 patients. }\end{array}$} \\
\hline Death & \multicolumn{2}{|c|}{ RR 0.98, 95\% Cl 0.83-1.16 } \\
\hline Nonfatal myocardial infarction & \multicolumn{2}{|c|}{ RR $0.98,95 \%$ Cl 0.83-1.16 } \\
\hline Nonfatal stroke & \multicolumn{2}{|c|}{ RR $0.78,95 \%$ Cl $0.63-0.98$} \\
\hline Heart failure & \multicolumn{2}{|c|}{ RR $0.54,95 \%$ Cl $0.37-0.81$} \\
\hline
\end{tabular}

\section{Beta blocker compared to other antihypertensive agents}

( 7 trials and $n=87,180$ patients, range mean age 60.4 to 76 years)

Composite cardiovascular outcome of death, nonfatal myocardial infarction or nonfatal stroke

Death

Nonfatal myocardial infarction

Nonfatal stroke

Heart failure

Beta blockers were associated with a higher risk of events than were other antihypertensive agents $(7,405$ events, RR $1.06,95 \% \mathrm{Cl} 1.01-1.10)$.

RR $1.05,95 \%$ Cl $0.99-1.11$
RR $1.06,95 \%$ Cl $0.94-1.20$
RR $1.18,95 \%$ Cl $1.07-1.30$
RR $0.98,95 \%$ Cl $0.87-1.11$

\section{Conclusion}

Beta blockers should not be considered first-line therapy for older hypertensive patients without another indication for these agents; however, in younger patients beta blockers are associated with a significant reduction in cardiovascular morbidity and mortality.

\section{Quality appraisal}

\section{Quality criteria for systematic reviews and meta- Author's analyses \\ Precise and accurately defined research question (e.g. PICO) \\ Well-defined selection criteria \\ Was an 'a priori' design provided? \\ Systematic literature research \\ Appropriate search strings, data bases and hand search \\ judgement \\ Yes \\ Yes \\ No \\ Yes \\ No \\ At least two reviewers for selecting retrieved studies Unclear}

\section{Support for judgement}

Criteria for considering studies are explicitly explained in the paper. The PICOS scheme can be applied

See above

There is no published protocol for this meta-analysis

Search method is illustrated

Only a PubMed search and a hand search were conducted. A very limited search string was used.

The review process, screening abstracts and reading full texts is not described. Two authors extracted outcome data from each trial independently. The inter observer kappa for trial inclusion was 0.94

Well documented process of selection of included Unclear studies (e.g. PRISMA flow diagram)

Quality of the studies documented and considered No for the synthesis of evidence

Was the conflict of interest stated?

Assessed publication bias

Heterogeneity statistically analysed

Quality of internal validity

Legend: $R C T$ randomized controlled trial, $A C E$ angiotensin-converting enzyme, $A R B$ angiotensin-receptor blockers, $B B$ Beta-blockers, $C C B$ calcium channel blockers, FU Follow up, TD Thiazide diuretic

$p=0.48)$, as well as Ogihara et al.: all-cause mortality: benidipine plus beta blocker vs. benidipine plus angiotensine receptor blocker HR 0.99 (0.54-1.82); benidipine plus beta blocker vs. benidipine plus thiazide diuretic HR 1.34 (0.69-2.60) [41].Testa et al. [46] found that older adults taking atenolol showed higher mortality 
Table 3 Summary of characteristics of the included studies

\begin{tabular}{|c|c|c|c|c|c|c|}
\hline $\begin{array}{l}\text { Author } \\
\text { year }\end{array}$ & $\begin{array}{l}\text { Type of } \\
\text { study }\end{array}$ & Aim & Intervention/control & Sample size & Follow-up & $\begin{array}{l}\text { Outcomes and } \\
\text { measurement tools if } \\
\text { applicable }\end{array}$ \\
\hline $\begin{array}{l}\text { Carlsson } \\
2014 \text { [43] }\end{array}$ & Cohort study & $\begin{array}{l}\text { To study mortality rates in } \\
\text { men and woman with } \\
\text { hypertension and AF } \\
\text { prescribes different } \\
\text { cardiovascular } \\
\text { pharmacotherapies } \\
\text { (time to death between } \\
\text { registration of AF } \\
\text { diagnosis and 31.12.2007) }\end{array}$ & $\begin{array}{l}\text { Antithrombotic drugs } \\
\text { alone: } \\
\text { - antiplatelet agents vs. } \\
\text { no treatment } \\
\text { - anticoagulants vs. no } \\
\text { treatment } \\
\text { - anticoagulants vs. } \\
\text { antiplatelets } \\
\text { Beta blocker: } \\
\text { - Selective vs. no } \\
\text { treatment } \\
\text { - non-elective vs. no } \\
\text { treatment } \\
\text { - non-selective vs. } \\
\text { selective } \\
\text { RAS-blocking agents: } \\
\text { - vs. no treatment } \\
\text { - ARBs vs. ACE inhibitors } \\
\text { - RAS blocking agents + } \\
\text { other vs. no treatment } \\
\text { - ARB + thiazide vs. ACE } \\
\text { inhibitor + thiazide } \\
\text { Calcium receptor- } \\
\text { blocking agents: } \\
\text { - Vessel selective vs. no } \\
\text { treatment } \\
\text { - Heart active vs. no } \\
\text { treatment } \\
\text { - Vessel selective vs. } \\
\text { heart active } \\
\text { - - Statins }\end{array}$ & $\begin{array}{l}n=5602 \\
4748 \text { aged } \geq 65 \text { y } \\
854 \text { aged }<65 \text { y }\end{array}$ & $\begin{array}{l}\text { Mean follow- } \\
\text { up } 3.4 \text { years }\end{array}$ & Mortality \\
\hline $\begin{array}{l}\text { Collier } \\
2011 \text { [42] }\end{array}$ & $\begin{array}{l}\text { Multicentre, } \\
\text { international } \\
\text { randomized } \\
\text { trial }\end{array}$ & $\begin{array}{l}\text { To evaluate the efficacy } \\
\text { and safety of an } \\
\text { amlodipine versus an } \\
\text { atenolol-based antihyper- } \\
\text { tensive regimen among } \\
\text { older ( } \geq 65 \text { years) and } \\
\text { younger ( }<65 \text { years) } \\
\text { patients. }\end{array}$ & $\begin{array}{l}\text { Atenolol-based (atenolol } \\
\pm \text { thiazide diuretic) } \\
\text { Amlodipine-based } \\
\text { (amlodipine } \pm \\
\text { perindopril) }\end{array}$ & $\begin{array}{l}n=19,257 \\
8137 \text { aged } \geq 65 \text { and } \\
11,020 \text { aged }<65\end{array}$ & $\begin{array}{l}\text { Median follow } \\
\text { up } 5.5 \text { years }\end{array}$ & $\begin{array}{l}\text { Nonfatal myocardial } \\
\text { infarction and fatal } \\
\text { coronary heart disease } \\
\text { and cardiovascular events }\end{array}$ \\
\hline $\begin{array}{l}\text { Coope } \\
1986[34]\end{array}$ & RCT & $\begin{array}{l}\text { To explore, if the } \\
\text { treatment of hypertension } \\
\text { in patients between the } \\
\text { ages of } 60 \text { and } 79 \text { years } \\
\text { old reduces the incidence } \\
\text { of stroke or coronary } \\
\text { disease or affects } \\
\text { cardiovascular or overall } \\
\text { mortality }\end{array}$ & $\begin{array}{l}\text { Intervention group: } \\
\text { Step 1: atenolol } 100 \mathrm{mg} \\
\text { If blood pressure control } \\
\text { was insufficient the } \\
\text { further treatment steps } \\
\text { were applied: } \\
\text { Step 2: bendrofluazide } \\
5 \mathrm{mg} \\
\text { Step 3: alphamethyldopa } \\
500 \mathrm{mg} \text { (and eventually } \\
\text { nifedipine retard } 20 \text { mg } \\
\text { or other antihypertensive } \\
\text { medication) } \\
\text { Control group: usual care }\end{array}$ & $\begin{array}{l}n=884 \\
\text { intervention group } \\
n=419 \\
\text { control group } \\
n=465\end{array}$ & $\begin{array}{l}\text { Mean follow } \\
\text { up } 4.4 \text { years } \\
\text { (range } 1-10 \text {, } \\
\text { SD not } \\
\text { reported) }\end{array}$ & $\begin{array}{l}\text { Primary outcomes: } \\
\text { myocardial infarction, } \\
\text { major strokes, minor } \\
\text { strokes, transient } \\
\text { ischaemic attacks, } \\
\text { death } \\
\text { Secondary outcomes: } \\
\text { Congestive heart failure } \\
\text { Heart failure } \\
\text { Atrial fibrillation } \\
\text { Clinical gout } \\
\text { Diabetes } \\
\text { Non- fatal cancer } \\
\text { Vertigo } \\
\text { Dizzy spells }\end{array}$ \\
\hline $\begin{array}{l}\text { Subgroup } \\
\text { analysis in } \\
\text { Coope } \\
1986[34]\end{array}$ & See above & $\begin{array}{l}\text { To analyse whether the } \\
\text { treatment of hypertension } \\
\text { in patients between the } \\
\text { ages of } 70 \text { and } 79 \text { years } \\
\text { old reduces the incidence } \\
\text { of all strokes }\end{array}$ & See above & Not stated & Not stated & $\begin{array}{l}\text { Incidence of all strokes } \\
\text { including major strokes, } \\
\text { minor strokes, transient } \\
\text { ischaemic attacks }\end{array}$ \\
\hline $\begin{array}{l}\text { Gelber } \\
2013 \text { [45] }\end{array}$ & $\begin{array}{l}\text { Prospective, } \\
\text { community- } \\
\text { based cohort } \\
\text { study }\end{array}$ & $\begin{array}{l}\text { To determine the } \\
\text { associations between } \\
\text { classes of antihypertensive } \\
\text { medication use and the }\end{array}$ & $\begin{array}{l}\text { No drug, BB alone, ACE } \\
\text { alone, Diuretic alone, } \\
\text { CCB alone, vasodilators } \\
\text { alone, BB \& } 1 \text { other, }\end{array}$ & $n=2197$ & $\begin{array}{l}\text { Median follow } \\
\text { up } 5.8 \text { years } \\
\text { (SD 5.1) }\end{array}$ & $\begin{array}{l}\text { Development of cognitive } \\
\text { impairment } \\
\text { CASI Score, a combination } \\
\text { of the }\end{array}$ \\
\hline
\end{tabular}


Table 3 Summary of characteristics of the included studies (Continued)

\begin{tabular}{|c|c|c|c|c|c|c|}
\hline & & $\begin{array}{l}\text { risk of cognitive } \\
\text { impairment among elderly } \\
\text { hypertensive men. }\end{array}$ & $\begin{array}{l}\text { Other drug } \\
\text { combinations }\end{array}$ & & & $\begin{array}{l}\text { Hasegawa Dementia } \\
\text { Screening Scale, the } \\
\text { Folstein Mini-Mental } \\
\text { State Examination, and } \\
\text { the Modified Mini-Mental } \\
\text { State Examination }\end{array}$ \\
\hline $\begin{array}{l}\text { Pepine } \\
2003 \text { [32] }\end{array}$ & $\mathrm{RCT}$ & $\begin{array}{l}\text { To test the hypothesis, } \\
\text { that the risk for adverse } \\
\text { outcomes in older people } \\
\text { with hypertension and } \\
\text { coronary artery disease } \\
\text { treated with a calcium } \\
\text { antagonist based strategy } \\
\text { or a non-calcium antagon- } \\
\text { ist (atenolol) based strat- } \\
\text { egy is equivalent. }\end{array}$ & $\begin{array}{l}\text { Step 1: either } \\
\text { calcium antagonist } \\
\text { group: } \\
240 \text { mg/d of verapamil } \\
\text { sustained release } \\
\text { or non-calcium antagon- } \\
\text { ist: } 50 \text { mg/d of atenolol } \\
\text { If target blood pressure } \\
\text { was not achieved, } \\
\text { further steps: } \\
\text { Step 2: } \\
\text { calcium antagonist } \\
\text { group additional } \\
\text { trandolapril } \\
\text { non-calcium antagonist } \\
\text { group additional } \\
\text { hydrochlorothiazide } \\
\text { Step 3: } \\
\text { dosage increase } \\
\text { Step 4: } \\
\text { calcium antagonist } \\
\text { group additional } \\
\text { hydrochlorothiazide } \\
\text { non-calcium antagonist } \\
\text { group additional } \\
\text { trandolapril. }\end{array}$ & $\begin{array}{l}n=22,576 \\
\text { Calcium antagonist } \\
\text { group, } n=11,267 \\
\text { Non-calcium antag- } \\
\text { onist group, } \\
n=11,309\end{array}$ & $\begin{array}{l}\text { Mean follow } \\
\text { up } 2.7 \text { years } \\
\text { (range } 1 \text { day } \\
\text { to } 5.4 \text { years, } \\
\text { SD not } \\
\text { reported) }\end{array}$ & $\begin{array}{l}\text { Primary outcome: } \\
\text { first occurrence of } \\
\text {-death (all cause) } \\
\text {-non-fatal myocardial } \\
\text { infarction } \\
\text {-non-fatal stroke } \\
\text { Secondary outcomes: } \\
\text { death (all cause), non-fatal } \\
\text { myocardial infarction, } \\
\text { non-fatal stroke, time to } \\
\text { most serious event } \\
\text { (ranked from death as } \\
\text { most serious, to myocar- } \\
\text { dial infarction, to stroke as } \\
\text { last serious), cardiovascular } \\
\text { death, angina, cardiovas- } \\
\text { cular hospitalizations, can- } \\
\text { cer, Alzheimer disease, } \\
\text { Parkinson, gastrointestinal } \\
\text { bleeding, blood pressure } \\
\text { control, new diagnosis of } \\
\text { diabetes, adverse } \\
\text { experiences. }\end{array}$ \\
\hline $\begin{array}{l}\text { Subgroup } \\
\text { analysis in } \\
\text { Pepine } \\
2003 \text { [32] }\end{array}$ & See above & See above & See above & $\begin{array}{l}\text { Subgroup > } 70 \text { years } \\
\text { old: calcium } \\
\text { antagonist group: } \\
n=3694 \text { non- } \\
\text { calcium antagonist } \\
\text { group: } n=3829\end{array}$ & See above & See above \\
\hline $\begin{array}{l}\text { Ruwald } \\
2012 \text { [41] }\end{array}$ & $\begin{array}{l}\text { Post hoc } \\
\text { analysis of a } \\
\text { double-blind, } \\
\text { double- } \\
\text { dummy ran- } \\
\text { domized trial }\end{array}$ & $\begin{array}{l}\text { To investigate the } \\
\text { influence of age on the } \\
\text { effects of losartan versus } \\
\text { atenolol-based antihyper- } \\
\text { tensive treatment }\end{array}$ & $\begin{array}{l}\text { Intervention: } \\
\text { losartan } 50 \text { mg } \\
\text { (step 2: + HCT } 12.5 \text { mg } \\
\text { HCT } \\
\text { step 3: losartan } \\
100 \text { mg + HCT } 12.5 \text { mg } \\
\text { step 4: losartan } \\
100 \text { mg + HCT 12.5- } \\
25 \text { mg HCT + another } \\
\text { antihypertensive } \\
\text { treatment) } \\
\text { Control: } \\
\text { atenolol } 50 \text { mg } \\
\text { (step 2: + HCT } 12.5 \text { mg } \\
\text { step 3: atenolol } \\
100 \text { mg + HCT } 12.5 \text { mg } \\
\text { step 4: atenolol } \\
100 \text { mg + HCT } 12.5- \\
25 \text { mg + another } \\
\text { antihypertensive } \\
\text { treatment) }\end{array}$ & $\begin{array}{l}n=9193 \\
n=4304<67 \text { years } \\
(46.8 \%) \\
n=4889 \geq 67 \text { years } \\
(53.2 \%)\end{array}$ & $\begin{array}{l}\text { Mean follow } \\
\text { up } 4.8 \text { years }\end{array}$ & $\begin{array}{l}\text { Primary outcome: } \\
\text { Composite of } \\
\text { cardiovascular death, } \\
\text { nonfatal stroke, nonfatal } \\
\text { Ml }\end{array}$ \\
\hline $\begin{array}{l}\text { Testa } \\
2014 \text { [44] }\end{array}$ & $\begin{array}{l}\text { Cross } \\
\text { sectional } \\
\text { study }\end{array}$ & $\begin{array}{l}\text { To evaluate long-term } \\
\text { mortality in hypertensive } \\
\text { older adults taking } \\
\text { atenolol }\end{array}$ & $\begin{array}{l}\text { No atenolol } \\
\text { (Intervention)/atenolol } \\
\text { (control) }\end{array}$ & $\begin{array}{l}972 \text { patients aged } \\
\geq 65 \text { with isolated } \\
\text { hypertension } \\
\text { mean age } 74.4 \pm 6.4\end{array}$ & $\begin{array}{l}\text { Follow up of } \\
12 \text { years from } \\
1992 \text { to } 2003 \\
\text { (not stated if } \\
\text { median or } \\
\text { mean follow } \\
\text { up) }\end{array}$ & $\begin{array}{l}\text { Taking atenolol showed a } \\
\text { greater mortality }(73.9 \% \text { vs } \\
55.0 \% ; p=0.047) \text { and } \\
\text { higher pulse arterial } \\
\text { pressure values ( } 74.7 \text { vs } \\
63.02 \text { mmHg, } p<0.001) \\
\text { than those not taking }\end{array}$ \\
\hline
\end{tabular}


Table 3 Summary of characteristics of the included studies (Continued)

Studies based on the COPE (Combination Therapy of Hypertension to Prevent Cardiovascular Events) trial

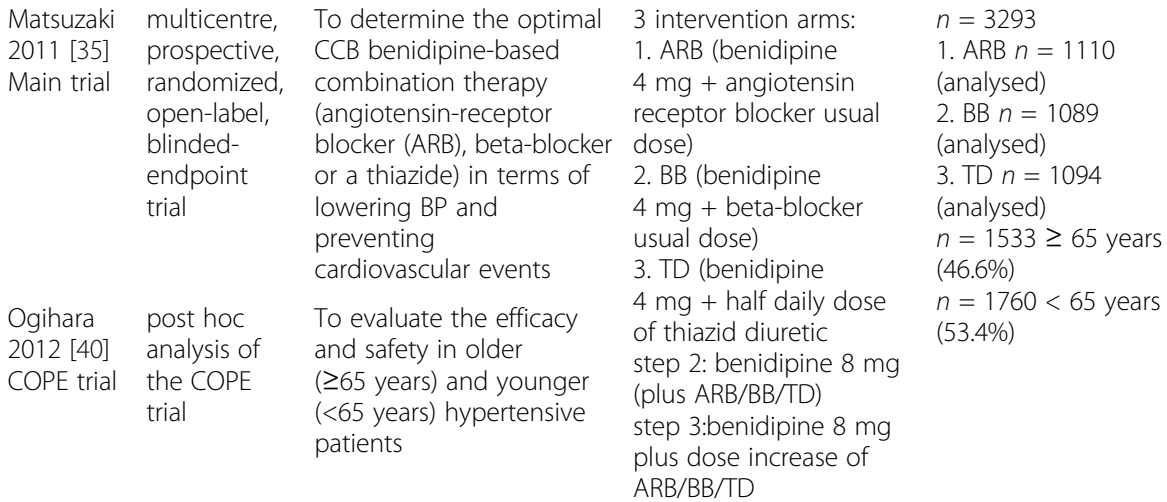

\author{
Median follow Primary outcome: \\ up 3.61 years composite of \\ cardiovascular morbidity \\ and mortality (sudden \\ death, fatal or non-fatal \\ stroke, fatal or non-fatal \\ myocardial infarction, \\ hospitalization due to \\ unstable angina, new \\ onset of \\ heart failure new onset or \\ worsening \\ of peripheral arterial \\ disease, new onset or \\ worsening of renal failure) \\ Secondary outcomes: \\ (1) All-cause mortality \\ (2) Death from \\ cardiovascular events \\ (3) Fatal and non-fatal car- \\ diovascular events \\ (4) New onset of diabetes \\ mellitus \\ (5) Safety (adverse events \\ and adverse drug \\ reaction) \\ (6) Nonfatal stroke \\ Hospitalization due to \\ heart failure (results on \\ outcome not reported)
}

\section{Studies based on the Medical Research Council (MRC) trial}

\begin{tabular}{|c|c|c|c|}
\hline $\begin{array}{l}\text { MRC } 1992 \\
{[33]} \\
\text { Main trial }\end{array}$ & $\mathrm{RCT}$ & $\begin{array}{l}\text { To establish whether } \\
\text { treatment with diuretic or } \\
\text { beta blocker in } \\
\text { hypertensive older adults } \\
\text { reduces risk of stroke, } \\
\text { coronary heart disease, } \\
\text { and death. }\end{array}$ & $\begin{array}{l}\text { Patients were } \\
\text { randomized to atenolol } \\
50 \mathrm{mg} \text { daily; } \\
\text { hydrochlorothiazide } \\
25 \mathrm{mg} \text { or } 50 \mathrm{mg} \text { plus } \\
\text { amiloride } 2.5 \mathrm{mg} \text { or } \\
5 \mathrm{mg} \text { (Moduretic } \odot \text { ) daily; } \\
\text { or placebo. }\end{array}$ \\
\hline $\begin{array}{l}\text { Bird } 1990 \\
{[36]}\end{array}$ & $\begin{array}{l}\mathrm{RCT}, \\
\text { secondary } \\
\text { analysis }\end{array}$ & $\begin{array}{l}\text { To explore if cognitive } \\
\text { dysfunction, manifested as } \\
\text { an increased incidence of } \\
\text { confusional states or } \\
\text { impaired concentration } \\
\text { and attention are } \\
\text { associated either with a } \\
\text { particular antihypertensive } \\
\text { regime or with the } \\
\text { reduction in blood } \\
\text { pressure level that it } \\
\text { induces. To explore if a } \\
\text { continuous reduction in } \\
\text { mildly elevated blood }\end{array}$ & $\begin{array}{l}\text { Patients were randomly } \\
\text { assigned to } \\
\text { hydrochlorothiazide } \\
25 \text { mg and } 2.5 \text { mg } \\
\text { amiloride, (Moduretic(C) } \\
\text { daily, atenolol } 50 \text { mg } \\
\text { daily, or placebo. }\end{array}$ \\
\hline
\end{tabular}

$n=4396$

Three groups

(a) potassium

sparing diuretic

regimen

(hydrochlorothiazide

Mean follow

up 5.8 years

25,355 patients

Strokes, coronary events, and deaths from all

years of

observation

tamiloride) $n=1081 \quad$ reported)

(b) beta blocker

atenolol $n=1102$

(c, d) matching

placebo tablets

$n=2213$

$n=2401$

hydrochlorothiazide

amiloride 24\%

atenolol 24\%

Placebo 52\%

\section{Results} reported at month 1 and 9 measured with the Nelson (median or mean follow up and SD not reported)

Cognitive tests: Verbal intelligence Adult Reading Test (NART), (performance ability) measured with the Ravens Matrice, part a \& b, Capacity to learn and remember new material measured with the Paired Associate Learning Test (PALT), 
Table 3 Summary of characteristics of the included studies (Continued)

pressure levels affect the

rate of change in

cognitive function.

Alertness and speed of reaction measured with the Trail-Making Test (TMT),

Depression with the selfrating Depression Questionnaire (DQ) Blood Pressure

\begin{tabular}{|c|c|c|c|c|c|c|}
\hline $\begin{array}{l}\text { Carr } 2012 \\
{[37]}\end{array}$ & $\begin{array}{l}\mathrm{RCT} \\
\text { secondary } \\
\text { analysis }\end{array}$ & $\begin{array}{l}\text { To assess the impact of } \\
\text { the blood pressure profile } \\
\text { on cardiovascular risk in } \\
\text { the Medical Research } \\
\text { Council (UK) elderly trial } \\
\text { and to investigate } \\
\text { whether the effects of } \\
\text { hypertensive drugs in } \\
\text { reducing event rates are } \\
\text { solely a } \\
\text { product of systolic } \\
\text { pressure reduction. }\end{array}$ & $\begin{array}{l}\text { Intervention:1. atenolol } \\
50 \mathrm{mg} \text { daily or } \\
2 . \text { hydrochlorothiazide } \\
25 \mathrm{mg} \\
(50 \mathrm{mg})+\text { amiloride } \\
2,5 \mathrm{mg}(5 \mathrm{mg}) \\
\text { Control: } \\
\text { Placebo }\end{array}$ & $\begin{array}{l}n=4396 \\
\text { atenolol: } n=1102 \\
\text { hydrochlorothiazide } \\
+ \text { amiloride: } \\
n=1081 \\
\text { hydrochlorothiazide } \\
+ \text { Placebo } n=2213\end{array}$ & $\begin{array}{l}\text { Mean follow } \\
\text { up } 5.8 \text { years }\end{array}$ & $\begin{array}{l}\text { Primary outcomes: } \\
\text { stroke } \\
\text { CHD } \\
\text { secondary outcomes: } \\
\text { association between BP } \\
\text { and outcomes }\end{array}$ \\
\hline $\begin{array}{l}\text { Lever } \\
1992 \text { [48] }\end{array}$ & $\begin{array}{l}\mathrm{RCT} \\
\text { secondary } \\
\text { analysis }\end{array}$ & $\begin{array}{l}\text { To study the effect of two } \\
\text { treatments for } \\
\text { hypertension on all-cause } \\
\text { mortality and morbidity } \\
\text { from cardiovascular } \\
\text { disease }\end{array}$ & see above & see above & see above & $\begin{array}{l}\text { Stroke } \\
\text { coronary events } \\
\text { all cardiovascular events } \\
\text { all-cause mortality } \\
\text { withdrawal/loss to FU } \\
\text { from treatment }\end{array}$ \\
\hline $\begin{array}{l}\text { Lever } \\
1993 \\
{[38]}\end{array}$ & $\begin{array}{l}\mathrm{RCT} \\
\text { secondary } \\
\text { analysis }\end{array}$ & $\begin{array}{l}\text { To determine whether } \\
\text { hypotensive drug } \\
\text { treatment in men and } \\
\text { women aged } 65-74 \\
\text { reduces stroke, CHD and } \\
\text { mortality }\end{array}$ & see above & see above & see above & $\begin{array}{l}\text { Stroke } \\
\text { coronary events } \\
\text { all cardiovascular events } \\
\text { all-cause mortality } \\
\text { change in blood pressure }\end{array}$ \\
\hline
\end{tabular}

Legend: $R C T$ randomized controlled trial, $A C E$ angiotensin-converting enzyme, $A R B$ angiotensin-receptor blockers, $B B$ Beta-blockers, $C C B$ calcium channel blockers, FU Follow up, TD Thiazide diuretic

rates than those not taking atenolol $(73.9 \%$ vs $55.0 \%$; $p=0.047)$. Cox regression analysis of this paper showed that atenolol use was predictive for long term mortality (HR 1.91; 95\% CI 1.04-4.31; $p=0.04$ ). Carlsson et al. [43] found that the prescription of beta 1 selective or non-selective beta blockers did not affect mortality other than no treatment (for numbers, see Table 6). Further, the prescription of non-selective beta blockers compared to beta 1 selective beta blockers was associated with lower mortality in sex-adjusted models HR 0.62 (95\% CI $0.41-0.95)$.

\section{Cardiovascular events - including stroke}

The analysis of the included group of studies of the meta-analysis [33] states that in older adults, beta blockers were associated with statistically significant reductions in nonfatal stroke (RR $0.78,95 \%$ CI 0.63-0.98) and heart failure (RR 0.54, 95\% CI 0.37-0.81) compared with placebo, but had no appreciable impact on rates of myocardial infarction (RR 0.98, 95\% CI 0.83-1.16). Compared to other antihypertensive agents, beta blockers were associated with significantly higher rates of nonfatal stroke (RR 1.18, 95\% CI 1.07-1.30), but not of heart failure (RR 0.98, 95\% CI 0.87-1.11) and myocardial infarction (RR 1.06, 95\% CI 0.94-1.20). The observational study of Collier et al. [44]compared an amlodipine- to a atenolol-based treatment and confirms these latter statements by showing that the amlodipine-based treatment reduced the relative risk of cardiovascular events more efficiently in older adults by $17 \%$ (HR 0.83 ; 95\% CI $0.75-0.91 ; p<0.01)$ and cardiovascular mortality by $23 \%$ (HR $0.77 ; 95 \%$ CI $0.63-0.94 ; p<0.01$ ). In this paper fatal and non-fatal stroke were also more efficiently reduced in the amlodipine-based treatment by 30\% (HR 0.70; 95\% CI 0.59-0.84; $p<0.01$ ). The MRC trial [35] found no significant reduction in cardiovascular endpoints comparing beta blocker treatment with placebo in older adults: stroke total: RR 0.84 (95\% CI $0.62-1.14, p=0.26)$, all cardiovascular events: RR 0.98 (95\% CI 0.82-1.18, $p=0.84$ ). Carr et al. [42], a secondary analysis of the MRC trial found evidence that atenolol does not perform as well as the hydrochlorothiazide plus amiloride (Moduretic (C) in terms of stroke prevention: Moduretic 41.6 number of events /100 patient years, beta blocker $50.8 \%$ and placebo $60.5 \%$ (statistical tests missing). Matsuzaki et al. [47] analysed the composite endpoint of cardiovascular mortality and morbidity (see Table 3 ) and found, that there were no statistical 
Table 4 Characteristics of the participants in the included studies

\begin{tabular}{|c|c|c|c|c|c|c|c|}
\hline $\begin{array}{l}\text { Author } \\
\text { year }\end{array}$ & $\begin{array}{l}\text { Setting / } \\
\text { country / } \\
\text { ethnicity }\end{array}$ & Male sex & $\mathrm{Age}^{\mathrm{a}}$ & Reported comorbidities & $\begin{array}{l}\text { Reported concomitant } \\
\text { medications }\end{array}$ & $\begin{array}{l}\text { Functional } \\
\text { status/ } \\
\text { Frailty level }\end{array}$ & Cognitive status \\
\hline $\begin{array}{l}\text { Carlsson } \\
2014 \text { [43] }\end{array}$ & Sweden & $50.14 \%$ & $\begin{array}{l}\text { Men: mean age } \\
72.3 \text { years, } \\
79 \% \geq 65 \text { years. } \\
\text { Woman: mean } \\
\text { age } 77.2 \text { years, } \\
92 \% \\
\geq 65 \text { years }\end{array}$ & $\begin{array}{l}\text { Diagnosis of both AF and } \\
\text { hypertension (inclusion } \\
\text { criteria), } \\
\text { coronary heart disease, } \\
\text { cerebrovascular diseases, } \\
\text { including intracranial } \\
\text { bleeding and peripheral } \\
\text { embolism, congestive heart } \\
\text { failure, DM II }\end{array}$ & $\begin{array}{l}\text { - Diuretic drugs (thiazides, } \\
\text { related agents, combined } \\
\text { formulations with other } \\
\text { drugs, loop diuretics, } \\
\text { potassium-saving diuretics) } \\
\text { - Beta blocker (B1- } \\
\text { selective, non-selective) } \\
\text { - Calcium receptor- } \\
\text { blocking agents (vessel-se- } \\
\text { lective, heart-selective) } \\
\text { - RAS-Blockers (ACE-inhibi- } \\
\text { tors, ARBs) } \\
\text { - Lipid-lowering drugs } \\
\text { (statins) } \\
\text { - antithrombotic drugs }\end{array}$ & $\begin{array}{l}\text { Not } \\
\text { reported }\end{array}$ & Not reported \\
\hline $\begin{array}{l}\text { Collier } \\
2011 \text { [44] }\end{array}$ & $\begin{array}{l}\text { UK, } \\
\text { Sweden, } \\
\text { Denmark, } \\
\text { Iceland, } \\
\text { Norway, } \\
\text { and Finland }\end{array}$ & $\begin{array}{l}\text { Patients } \geq 65 \\
\text { Amlodipine- } \\
\text { based } \\
\text { regimen } \\
73.6 \% \\
\text { Atenolol- } \\
\text { based } \\
\text { regimen } \\
73.5 \%\end{array}$ & $\begin{array}{l}\text { Patients } \geq 65 \\
\text { Amlodipine- } \\
\text { based regimen } \\
71.1 \text { (4.0) } \\
\text { Atenolol-based } \\
\text { regimen } \\
71.1 \text { (4.0) }\end{array}$ & $\begin{array}{l}\text { Type } 2 \text { diabetes mellitus, ECG } \\
\text { abnormalities (not LVH), LVH } \\
\text { (on ECG or ECHO), Peripheral } \\
\text { vascular disease }\end{array}$ & $\begin{array}{l}\text { No previous } \\
\text { antihypertensive use, } \mathrm{n} \\
(\%): \\
\text { Amlodipine-based } \\
\text { regimen } \\
681 \text { (16.8) } \\
\text { Atenolol-based regimen } \\
677(16.5) \\
\text { Aspirin use, n (\%): } \\
\text { Amlodipine-based } \\
\text { regimen } \\
1066(26.4) \\
\text { Atenolol-based regimen } \\
1046(25.5)\end{array}$ & $\begin{array}{l}\text { Not } \\
\text { reported }\end{array}$ & Not reported \\
\hline $\begin{array}{l}\text { Coope } \\
1986 \text { [37] }\end{array}$ & $\begin{array}{l}13 \text { general } \\
\text { practices in } \\
\text { England } \\
\text { and Wales }\end{array}$ & $\begin{array}{l}\text { Intervention } \\
\text { group: } \\
\text { 29.0\% } \\
\text { Control } \\
\text { group: } \\
\text { 32.0\% }\end{array}$ & $\begin{array}{l}\text { Intervention } \\
\text { group: } 68.7 \\
\text { (5.2) years } \\
\text { Control group: } \\
68.8 \text { (5.1) years }\end{array}$ & $\begin{array}{l}\text { Intervention group } \\
\text { Left ventricular hypertrophy } \\
\text { on ECG: } n=8 \\
\text { Cardiac enlargement on } \\
\text { chest XRay: } n=22 \\
\text { Control group } \\
\text { Left ventricular hypertrophy } \\
\text { on ECG: } n=11 \\
\text { Cardiac enlargement on } \\
\text { chest XRay: } n=21\end{array}$ & Not stated & Not stated & Not stated \\
\hline $\begin{array}{l}\text { Subgroup } \\
\text { analysis in } \\
\text { Coope } \\
1986[37]\end{array}$ & See above & $\begin{array}{l}29.1 \% \\
\text { (based on } \\
\text { own } \\
\text { calculations) }\end{array}$ & 70-79 years & Not stated & See above & See above & See above \\
\hline $\begin{array}{l}\text { Gelber } \\
2013 \text { [45] }\end{array}$ & $\begin{array}{l}\text { Hawaii, } \\
\text { USA } \\
\text { (Japanese } \\
\text { men) }\end{array}$ & $100 \%$ & $\begin{array}{l}\text { Overall mean } \\
\text { age: } 77 \\
\text { No drug } 77.2 \\
\text { (4.2) } \\
\text { BB alone } 76.2 \\
\text { (3.8) } \\
\text { ACE alone } 76.7 \\
\text { (4.1) } \\
\text { Diuretic alone } \\
77.4 \text { (4.0) } \\
\text { CCB alone } 76.9 \\
\text { (4.0) } \\
\text { Vasodilators } \\
\text { alone } 76.8 \text { (3.6) } \\
\text { BB \& } 1 \text { other } \\
76.5 \text { (3.6) } \\
\text { Other drug } \\
\text { combi-nation } \\
77.0 \text { (4.0) }\end{array}$ & $\begin{array}{l}\text { Type } 2 \text { diabetes mellitus, CVD } \\
\text { (defined as history of } \\
\text { myocardial infarction, angina } \\
\text { and other coronary heart } \\
\text { disease or stroke), APOE }\end{array}$ & Not reported & $\begin{array}{l}\text { Not } \\
\text { reported }\end{array}$ & $\begin{array}{l}\text { BB use was not } \\
\text { significantly } \\
\text { associated with } \\
\text { a } \\
\text { lower risk of } \\
\text { cognitive } \\
\text { impairment, } \\
\text { suggesting that } \\
\text { medication class } \\
\text { may be less } \\
\text { relevant if the } \\
\text { SBP is not } \\
\text { adequately } \\
\text { controlled. }\end{array}$ \\
\hline
\end{tabular}


Table 4 Characteristics of the participants in the included studies (Continued)

\begin{tabular}{|c|c|c|c|c|c|c|c|}
\hline $\begin{array}{l}\text { Pepine } \\
2003 \text { [34] }\end{array}$ & $\begin{array}{l}862 \text { primary } \\
\text { care sites in } \\
14 \\
\text { countries } \\
\text { worldwide } \\
\text { Calcium } \\
\text { antagonist } \\
\text { group (\%): } \\
\text { White 48.5 } \\
\text { Hispanic } \\
35.7 \\
\text { Black 13.4 } \\
\text { Asian 0.6 } \\
\text { Other/ } \\
\text { multiracial } \\
1.9 \\
\text { Non- } \\
\text { calcium } \\
\text { antagonist } \\
\text { group (\%): } \\
\text { White 48.3 } \\
\text { Hispanic } \\
35.6 \\
\text { Black } 35.6 \\
\text { Asian 0.8 } \\
\text { Other/ } \\
\text { multiracial } \\
1.9\end{array}$ & $\begin{array}{l}\text { Calcium } \\
\text { antagonist } \\
\text { group (\%): } \\
48.1 \\
\text { Non-calcium } \\
\text { antagonist } \\
\text { group (\%): } \\
47.7\end{array}$ & $\begin{array}{l}\text { Calcium } \\
\text { antagonist } \\
\text { group: } 66,0 \\
\text { (9.7) years } \\
\text { Non-calcium } \\
\text { antagonist } \\
\text { group: } 66,1 \\
\text { (9.8) years }\end{array}$ & $\begin{array}{l}\text { Calcium antagonist group } \\
(\%) \text { : } \\
\text { Myocardial infarction } 32.1 \\
\text { Prior myocardial infarction or } \\
\text { abnormal angiogram } 52.6 \\
\text { Angina pectoris } 66.2 \\
\text { CABG } \geq 1 \text { month ago } 15.5 \\
\text { PCI } \geq 1 \text { month ago } 15.2 \\
\text { CABG or PCI } 27.3 \\
\text { Stroke } 5.3 \\
\text { Left ventricular hypertrophy } \\
21.5 \\
\text { Unstable angina } \geq 1 \text { month } \\
\text { ago } 11.4 \\
\text { Arrhythmia } 7.1 \\
\text { Heart failure class } 1 \text { I-III } 5.5 \\
\text { Peripheral vascular disease } \\
11.9 \\
\text { Diabetes } 28.1 \\
\text { Hypercholesterolemia } 55.9 \\
\text { Renal impairment } 1.9 \\
\text { Cancer } 3.5 \\
\text { Non-calcium antagonist } \\
\text { group (\%): } \\
\text { Myocardial infarction } 31.8 \\
\text { Prior myocardial infarction or } \\
\text { abnormal angiogram } 53.3 \\
\text { Angina pectoris } 67 \\
\text { CABG } \geq 1 \text { month ago } 16.1 \\
\text { PCI } 1 \text { month ago } 14.7 \\
\text { CABG or PCl } 27.3 \\
\text { Stroke } 5.0 \\
\text { Left ventricular hypertrophy } \\
22.3 \\
\text { Unstable angina } \geq 1 \text { month } \\
\text { ago } 11.5 \text { Arrhythmia } 7.1\end{array}$ & $\begin{array}{l}\text { Calcium antagonist group } \\
(\%) \text { : } \\
\text { Aspirin@ or other } \\
\text { antiplatelet inhibitors } 57 \\
\text { NSAIDs } 17.6 \\
\text { Antidiabetic medication } \\
22.1 \\
\text { Any lipid-lowering agent } \\
36.8 \\
\text { Nitrates } 35.4 \\
\text { Potassium supplement } 6.9 \\
\text { Hormone replacement } \\
17.7 \\
\text { Non-calcium antagonist } \\
\text { group: } \\
\text { Aspirin@ or other } \\
\text { antiplatelet inhibitors: } \\
56.4 \\
\text { NSAIDs: } 17.9 \\
\text { Antidiabetic medication: } \\
22.9 \\
\text { Any lipid-lowering agent: } \\
36.6 \\
\text { Nitrates: } 36.6 \\
\text { Potassium supplement } 6.9 \\
\text { Hormone replacement } \\
18.5\end{array}$ & Not stated & $\overline{\text { Not stated }}$ \\
\hline & & & & $\begin{array}{l}\text { Heart failure class II-III } 5.6 \\
\text { Peripheral vascular disease } \\
12.0 \\
\text { Diabetes } 28.6 \\
\text { Hypercholesterolemia } 55.6 \\
\text { Renal impairment } 1.9 \\
\text { Cancer } 3.3\end{array}$ & & & \\
\hline $\begin{array}{l}\text { Subgroup } \\
\text { analysis in } \\
\text { Pepine } \\
2003[34]\end{array}$ & Not stated & Not stated & $>70$ years & Not stated & Not stated & Not stated & Not stated \\
\hline $\begin{array}{l}\text { Ruwald } \\
2012 \text { [40] }\end{array}$ & $\begin{array}{l}\text { Denmark, } \\
\text { Finland, } \\
\text { Iceland, } \\
\text { Norway, } \\
\text { Sweden, } \\
\text { and UK, }\end{array}$ & $\begin{array}{l}\text { 45-66 years: } \\
\text { losartan: } \\
51.2 \% \\
\text { atenolol: } \\
50.6 \% \\
67-83 \text { years: } \\
\text { losartan: } \\
41.3 \% \\
\text { atenolol: } \\
42.0 \%\end{array}$ & $\begin{array}{l}\text { overall mean } \\
\text { age } 67 \text { years } \\
46.8 \% \\
<66 \text { years } \\
53.2 \% \\
\geq 67 \text { years }\end{array}$ & $\begin{array}{l}\text { Any vascular disease: } 25 \% \\
\text { (Coronary heart disease } 16 \% \text {, } \\
\text { cerebrovascular disease } 8 \% \text {, } \\
\text { peripheral vascular disease } \\
6 \% \text {, atrial fibrillation: } 4 \% \\
\text { diabetes: } 13 \% \\
\text { isolated systolic hypertension: } \\
14 \%\end{array}$ & Not reported & $\begin{array}{l}\text { Not } \\
\text { reported }\end{array}$ & Not reported \\
\hline $\begin{array}{l}\text { Testa } \\
2014 \text { [46] }\end{array}$ & $\begin{array}{l}\text { Campania/ } \\
\text { Southern } \\
\text { Italy }\end{array}$ & $41 \%$ & $\begin{array}{l}\text { Mean age } 74.4 \\
( \pm 6.4) \\
93 \% \geq 65 \mathrm{y} .\end{array}$ & $\begin{array}{l}\text { Hypertension (inclusion } \\
\text { criteria) } \\
\text { Diabetes, chronic renal failure, } \\
\text { AF }\end{array}$ & $\begin{array}{l}\text { ACE-inhibitors, diuretics, } \\
\text { hypolipidemic drugs }\end{array}$ & $\begin{array}{l}\text { BADL (basic } \\
\text { activities of } \\
\text { daily living) } \\
\text { GDS } \\
\text { (geriatric } \\
\text { depression } \\
\text { scale) }\end{array}$ & $\begin{array}{l}\text { MMSE: } \\
\text { Atenolol vs. no } \\
\text { Atenolol } 24.9 \text { vs. } \\
25.1(p=0.841)\end{array}$ \\
\hline
\end{tabular}


Table 4 Characteristics of the participants in the included studies (Continued)

\begin{tabular}{|c|c|c|c|c|c|c|c|}
\hline \multicolumn{8}{|c|}{ Studies based on the COPE (Combination Therapy of Hypertension to Prevent Cardiovascular Events) trial } \\
\hline $\begin{array}{l}\text { Author } \\
\text { year }\end{array}$ & $\begin{array}{l}\text { Setting / } \\
\text { country / } \\
\text { ethnicity }\end{array}$ & Male sex & Age & Reported comorbidities & $\begin{array}{l}\text { Reported concomitant } \\
\text { medications }\end{array}$ & $\begin{array}{l}\text { Functional } \\
\text { status/ } \\
\text { Frailty } \\
\text { level }\end{array}$ & $\begin{array}{l}\text { Cognitive } \\
\text { status }\end{array}$ \\
\hline $\begin{array}{l}\text { COPE trial } \\
\text { Matsuzaki } \\
{[47]} \\
\text { Main trial }\end{array}$ & Japan & $\begin{array}{l}\text { Overall: } \\
\text { benidipine } \\
\text { plus } \\
\text {-ARB: } 51 \% \\
\text {-BB: } 50.5 \% \\
\text {-TD: } 50.5 \%\end{array}$ & $\begin{array}{l}\text { Benidipine plus } \\
\text {-ARB: } 63.0(10.6) \\
\text {-BB: } 63.2(10.8) \\
\text {-TD: } 63.1(10.8)\end{array}$ & $\begin{array}{l}\text { ARB/BB/TD groups: } \\
\text { Overall: } \\
\text { previous casdiovascular } \\
\text { disease: } 13 \% / 11.4 \% / 12.5 \% \\
\text { Arrhythmia: } 2.7 \% / 3.0 \% / 2.4 \% \\
\text { Diabetes: } 13.9 \% / 14.2 \% / 14.4 \%\end{array}$ & $\begin{array}{l}\text { ARB/BB/TD groups: } \\
\text { Overall: } \\
\text { Antiplatelet agents: } 8.9 \% / \\
6.8 \% / 7.3 \% \\
\text { Lipid-lowering agents: } \\
\text { 21.1\%/20.4\%/21.2\% } \\
\text { Antidiabetic agents: } 6.9 \% / \\
\text { 7.3\%/7.2\% }\end{array}$ & $\begin{array}{l}\text { Not } \\
\text { reported }\end{array}$ & Not reported \\
\hline $\begin{array}{l}\text { Ogihara } \\
\text { [41] }\end{array}$ & & $\begin{array}{l}\geq 65 \text { years: } \\
\text {-ARB: } 43.6 \% \\
\text {-BB: } 43.8 \% \\
\text {-TD: } 42.3 \%\end{array}$ & $\begin{array}{l}46.6 \% \text { aged } \\
\geq 65 \text { years } \\
\text { ( } \geq 65 \text { years: } \\
\text { mean age } \\
72.6 \text { years })\end{array}$ & $\begin{array}{l}\geq 65 \text { years: } \\
\text { previous cardiovascular } \\
\text { disease: } 18.3 \% \\
\text { stroke: } 4.4 \% \\
\text { angina pectoris: } 4.8 \% \\
\text { Ml: } 1.05 / 0.7 \% / 1.2 \% \\
\text { Arrhythmia: } 3.6 \% / 3.9 \% / 3.6 \% \\
\text { Diabetes: } 16.5 \% / 16.5 \% / 16.8 \%\end{array}$ & $\begin{array}{l}\geq 65 \text { years: } \\
\text { Antiplatelet agents: } 13.7 \% / \\
\text { 10.7\%/11.8\% } \\
\text { Lipid-lowering agents: } \\
\text { 23.1\%/21.7\%/20.4\% } \\
\text { Antidiabetic agents: } 8.6 \% / \\
\text { 9.2\%/9.0\% }\end{array}$ & & \\
\hline \multicolumn{8}{|c|}{ Studies based on the Medical Research Council (MRC) trial } \\
\hline $\begin{array}{l}\text { Author } \\
\text { year }\end{array}$ & $\begin{array}{l}\text { Setting / } \\
\text { country / } \\
\text { ethnicity }\end{array}$ & Male sex & Age & Reported comorbidities & $\begin{array}{l}\text { Reported concomitant } \\
\text { medications }\end{array}$ & $\begin{array}{l}\text { Functional } \\
\text { status/ } \\
\text { Frailty } \\
\text { level }\end{array}$ & $\begin{array}{l}\text { Cognitive } \\
\text { status }\end{array}$ \\
\hline $\begin{array}{l}\text { MRC } 1992 \\
{[35]} \\
\text { Main trial }\end{array}$ & $\begin{array}{l}226 \text { general } \\
\text { practices in } \\
\text { England, } \\
\text { Scotland, } \\
\text { and Wales }\end{array}$ & $\begin{array}{l}\text { Diuretic } \\
42.0 \% \\
\text { Beta blocker } \\
41,0 \% \\
\text { Placebo } \\
42,0 \%\end{array}$ & $\begin{array}{l}\text { Range } 65- \\
74 \text { years. }\end{array}$ & Not stated & Not stated & Not stated & Not stated \\
\hline $\begin{array}{l}\text { Bird } 1990 \\
{[36]}\end{array}$ & see above & $41.0 \%$ & $\begin{array}{l}\text { Mean }(S D) 70.3 \\
(2.7) \text { years }\end{array}$ & see above & see above & see above & see above \\
\hline $\begin{array}{l}\text { Carr } 2012 \\
{[42]}\end{array}$ & see above & $42 \%$ & $\begin{array}{l}\text { Mean age } \\
70.3 \text { years } \\
\text { Placebo } 70.3 \\
\text { Diuretic } 70.3 \\
\text { b-blocker } 70.4\end{array}$ & see above & see above & see above & see above \\
\hline $\begin{array}{l}\text { Lever } \\
1992[48]\end{array}$ & see above & see above & see above & see above & see above & see above & see above \\
\hline $\begin{array}{l}\text { Lever } \\
1993[39]\end{array}$ & see above & see above & see above & see above & see above & see above & see above \\
\hline
\end{tabular}

Legend: $C A B G$ coronary artery bypass graft, $P C l$ percutaneous coronary interventions, $S D$ standard deviation, ${ }^{a}$ Mean age (SD) years, ${ }^{b}$ Non Steroid Anti Inflammatory Drugs, $A P O E$ Apolipoprotein $\mathrm{E}, A R B$ angiotensin-receptor blocker, $B B$ beta-blocker, $C V D$ cardiovascular disease, MI Myocardial infarction, GDS Geriatric Depression Scale, TD: thiazide diuretic

significant differences regarding the analyses containing beta blockers: beta blocker compared to angiotensin receptor blocker HR 1.22 (95\% CI $0.80-1.85, p=0.3372$ ) and beta blocker compared to thiazide diuretic HR 1.54 (95\% CI 0.98-2.41, $p=0.0567$ ).

Regarding the endpoint of total stroke, Coope et al. [37] analysed data comparing beta blocker treatment to no treatment in a subgroup analysis involving older adults $>70$ and found a similar reduction in both groups. They further state that the sample was not large enough for the differences to be significant (numbers were not available from the paper). Ogihara et al. [41] states that benidipine plus beta blocker reduced fatal and non-fatal stroke less than benidipine plus thiazide diuretics (HR 2.74; 95\% CI 1.08-6.96).

\section{Cognitive impairment/status}

Bird [36], a secondary analysis of the the MRC trial [35] found that beta blocker treatment of moderate raised blood pressure in older adults did not impair cognitive function or produce symptoms or behaviour changes, which would cause concern in confidants of treated patients. Gelber et al. [45] found that beta blocker use as the sole 
Table 5 Summary of study findings of the meta-analysis (subgroup analysis)

\begin{tabular}{|c|c|}
\hline Author year & Objective \\
\hline Kahn 2006 [33] & $\begin{array}{l}\text { To explore the efficacy (stroke, myocardial infarction and death) of beta blockers } \\
\text { in "younger" }<60 \text { years) and "older" ( } \geq 60 \text { years) patients. }\end{array}$ \\
\hline \multicolumn{2}{|l|}{ Results: } \\
\hline \multicolumn{2}{|c|}{ 1. Beta blockers compared to placebo or no treatment ${ }^{\mathbf{1}}$ ( 5 trials and $n=8019$ patients, range mean age 65 to 75.7 years) } \\
\hline $\begin{array}{l}\text { Primary outcome: composite cardiovascular outcome of death, } \\
\text { nonfatal myocardial infarction or nonfatal stroke }\end{array}$ & $\begin{array}{l}\text { Beta blockers' benefits were not found in trials enrolling older patients } \\
\text { RR } 0.89,95 \% \mathrm{Cl} 0.75-1.05 \text {, based on } 1115 \text { events in } 8019 \text { patients. }\end{array}$ \\
\hline Death & RR $0.91,95 \%$ Cl $0.74-1.12$ \\
\hline Nonfatal myocardial infarction & RR $0.98,95 \%$ Cl 0.83-1.16 \\
\hline Nonfatal stroke & RR $0.78,95 \%$ Cl $0.63-0.98$ \\
\hline Heart failure & RR $0.54,95 \%$ Cl $0.37-0.81$ \\
\hline \multicolumn{2}{|c|}{ 2. Beta blocker compared to other antihypertensive agents ${ }^{1}$ ( 7 trials and $n=87,180$ patients, range mean age 60.4 to 76 years) } \\
\hline $\begin{array}{l}\text { Primary outcome: composite cardiovascular outcome of death, } \\
\text { nonfatal myocardial infarction or nonfatal stroke }\end{array}$ & $\begin{array}{l}\text { Beta blockers were associated with a higher risk of events than were other } \\
\text { antihypertensive agents ( } 7405 \text { events, RR 1.06, 95\% Cl 1.01-1.10). }\end{array}$ \\
\hline Death & RR $1.05,95 \%$ Cl 0.99-1.11 \\
\hline Nonfatal myocardial infarction & RR $1.06,95 \%$ Cl 0.94-1.20 \\
\hline Nonfatal stroke & RR $1.18,95 \%$ Cl 1.07-1.30 \\
\hline Heart failure & RR $0.98,95 \%$ Cl $0.87-1.11$ \\
\hline
\end{tabular}

Legend: $R R$ relative risk, $C l$ confidence interval, ${ }^{1} \mathrm{p}$-values not reported

antihypertensive medication was associated with a lower risk of developing cognitive impairment defined as a CASI (Cognitive Abilities Screening Instrument) score < 74) compared with untreated men (incidence rate ratio IRR 0.69; 95\% CI 0.50-0.94). Beta blocker use was also associated with a trend toward a decreased risk of cognitive decline (defined as $\mathrm{a} \geq 9$ point decrease in CASI score): adjusted analysis IRR 0.78 (95\% CI 0.61-1.00) for beta blocker use alone; IRR 0.81 (95\% CI 0.64-1.03) for beta blocker in combination with other drugs. None of the other drug categories was significantly associated with cognitive decline.

\section{Adverse events}

The Matsuzaki trial [47] reported about adverse events. The number of patients who discontinued the trial because of serious adverse events was 12 (1.1\%), 11 (1.0\%), and $11(1.0 \%)$ in the benidipine-ARB, benidipine-bblocker, and benidipine-thiazide groups, respectively. The percentage of adverse events was similar among the treatment groups: 505 (45.5\%), 495 (45.5\%), and 522 (47.7\%) of the patients reported adverse events. Bradycardia (benidipine-b-blocker, $p<0.0001$ ) and vertigo (benidipine-bblocker and benidipine- thiazide, $p=0.0188$ ) occurred more frequently in the beta blocker group.

\section{Risk of bias - meta-analysis}

A summary of risk of bias in the meta-analysis [33] is presented in Table 2. The main limitations contributing to a risk of bias were related to methodology issues. The literature search was done without using appropriate search strings and only two data bases were used, namely
MEDLINE and the Cochrane library. A quality appraisal of included studies was lacking. The review process was unclear and a list of excluded studies with reason for exclusion was missing. Risk of publication bias was not assessed.

\section{Risk of bias - Clinical trials}

A summary of risk of bias for intervention studies is presented in Fig. 2. The main limitations contributing to risk of bias were related to the design (e.g. inadequate randomisation, blinding, attrition bias and selective reporting). Other potential sources of bias were related to intentionto-treat analysis, contamination bias, sample size and power calculation. In two studies a high crossover rate between groups was registered [34, 37]. Detailed information of the risk of bias is available in Additional file 4.

\section{Risk of bias - Observational studies}

Quality appraisal of the four observational studies is shown in Table 7. Three studies can be judged as being of fairly good quality. One study reported on most of the CASP items insufficiently and cannot be considered of high quality.

\section{Additional references of interest for the development of recommendations}

We found nine additional references that were taken into consideration for the development of the recommendations: three Cochrane reviews [17, 49, 50], one update of a Cochrane review [22], one meta-analysis [33], two evidence based recommendation paper [51,52], one evidence based guideline [38] and one randomized controlled trial 
Table 6 Summary of study findings

\begin{tabular}{|c|c|c|}
\hline Authors year & \multicolumn{2}{|l|}{ Findings } \\
\hline \multirow[t]{4}{*}{ Carlsson 2014 [43] } & \multicolumn{2}{|c|}{$\begin{array}{l}\text { Prescription of selective or non-selective beta blockers did not affect mortality other than no treatment. Prescription of } \\
\text { non-selective beta blockers was associated with lower mortality in sex-adjusted models } \\
\text { Full regression model of the whole study sample adjusted for sex and all other covariates: } \\
\text { non-selective beta blockers vs. beta 1-selective beta blockers HR } 0.62 \text { ( } 95 \% \mathrm{Cl}: 0.41-0.95) \text {. }\end{array}$} \\
\hline & $\begin{array}{l}\text { men aged } \geq 80 \text { y }(H R(95 \% C l)) \text { : } \\
\text { beta } 1 \text {-selective vs. no treatment: } \\
\text { Model A: } 1.01(0.60-1.70) \text { Model B: } 1.09(0.64- \\
\text { 1.85) } \\
\text { non-selective vs. no treatment: } \\
\text { Model A: } 0.47 \text { (0.14-1.64) Model B: } 0.53(0.15- \\
\text { 1.88) } \\
\text { non-selective vs. beta 1-selective } \\
\text { Model A: } 0.42 \text { (0.13-1.37) Model B: } 0.39(0.12- \\
1.25)\end{array}$ & $\begin{array}{l}\text { men all ages (HR }(95 \% \mathrm{Cl}) \text { ): } \\
\text { beta 1-selective vs. no treatment: } \\
\text { Model A: } 0.99(0.69-1.40) \text { Model B: } 1.12(0.77-1.59) \\
\text { non-selective vs. no treatment: } \\
\text { Model A: } 0.55 \text { (0.28-1.08) Model B: } 0.68(0.34-1.36) \\
\text { non-selective vs. beta 1-selective } \\
\text { Model A. } 0.57 \text { (0.32-1.05) Model B: } 0.54(0.29-1.01)\end{array}$ \\
\hline & $\begin{array}{l}\text { women aged } \geq 80 \text { y(HR }(95 \% \mathrm{Cl})) \text { : } \\
\text { beta 1-selective vs. no treatment: } \\
\text { Model A: } 0.87(0.58-1.31) \text { Model B: } 0.90(0.59- \\
\text { 1.38) } \\
\text { non-selective vs. no treatment: } \\
\text { Model A: } 0.77(0.36-1.64) \text { Model B: } 0.76(0.35- \\
\text { 1.64) } \\
\text { non-selective vs. beta 1-selective } \\
\text { Model A:0.88 (0.44-1.76) Model B: } 0.86(0.41- \\
1.75)\end{array}$ & $\begin{array}{l}\text { women all ages }(H R(95 \% C l)) \text { : } \\
\text { beta 1-selective vs. no treatment } \\
\text { Model A: } 0.85(0.60-1.20) \text { Model B: } 0.88(0.62-1.25) \\
\text { non-selective vs. no treatment } \\
\text { Model A: } 0.62(0.33-1.17) \text { Model B. } 0.66(0.34-1.25) \\
\text { non-selective vs. beta 1-selective } \\
\text { Model A: } 0.76(0.43-1.35) \text { Model B: } 0.73(0.41-1.31)\end{array}$ \\
\hline & \multicolumn{2}{|c|}{$\begin{array}{l}\text { Model A (prospensity score age group, cardiovascular comorbidity (diabetes, CHD, CHF and CVDs), educational level and } \\
\text { marital status) } \\
\text { Model B (propensity score including all variables in Model A and all the antihypertensive drugs, antithrombotics and statin } \\
\text { (except the studied drug class). }\end{array}$} \\
\hline Collier 2011 [44] & \multicolumn{2}{|c|}{$\begin{array}{l}\text { Compared with the atenolol-based regimen, the amlodipine-based regimen reduced: } \\
\text { - the relative risk of cardiovascular events more effectively in both age groups: by } 17 \% \text { in patients } \geq 65 \text { years (hazard ratio: } \\
0.83 ; 95 \% \mathrm{Cl} 0.75,0.91 ; P<0.01 \text { ) and } 15 \% \text { in patients }<65 \text { years (hazard ratio } 0.85 ; 95 \% \mathrm{Cl} 0.78,0.95 ; P<0.01 \text { ) } \\
\text { - cardiovascular mortality by } 23 \% \text { in the older group (hazard ratio } 0.77 ; 95 \% \mathrm{Cl} 0.63,0.94 ; P<0.01 \text { ) and by } 24 \% \text { in the } \\
\text { younger group (hazard ratio } 0.76 ; 95 \% \mathrm{Cl} 0.58,1.00 ; P=0.05 \text { ) } \\
\text { - fatal and nonfatal stroke by } 30 \% \text { in the older group (hazard ratio } 0.70 ; 95 \% \mathrm{Cl} 0.59,0.84 ; P<0.01 \text { ) and by nonsignificant } \\
9 \% \text { in the younger group (hazard ratio } 0.91 ; 95 \% \mathrm{Cl} 0.71,1.15 ; P=0.42 \text { ) } \\
\text { - - significant in total of coronary endpoints, nonfatal MI (excluding silent } \mathrm{MI} \text { ) and fatal } \mathrm{CHD} \text { in the younger, but not in the } \\
\text { older group }\end{array}$} \\
\hline
\end{tabular}

Coope 1986 [37] Overall no significant difference in the total mortality was found neither in treatment nor in the control group. The rate of all deaths in the intervention group was 0.97 of that in the control group $(95 \% \mathrm{Cl} 0.70-1.42)$. The rate of fatal stroke in the intervention group was 0.30 of that in the control group $(95 \% \mathrm{Cl} 0.11-0.84) p<0.025$. Rate of all stroke in the intervention group was 0.58 of that in the control group $(95 \% \mathrm{Cl} 0.35-0.96) p<0.03$.

The subgroup analysis of patients by age (70-79 years) showed a similar reduction in total stroke in both groups, but the study was not large enough for these differences to be significant.

Gelber 2013 [45] Beta blocker use as the sole antihypertensive medication was associated with a lower risk of developing cognitive impairment (defined as a CASI [Cognitive Abilities Screening Instrument]) score < 74) compared with untreated men (IRR 0,69; 95\% Cl 0,50-0,94). Non- beta blocker drug combinations were also associated with a reduced risk (IRR 0,78; 95\% Cl $0,62-0,98)$

Cognitive decline (defined as a $\geq 9$ point decrease in CASI score) occurred in 1167 men (53.1\%). Beta blocker use was also associated with a trend toward a decreased risk of cognitive decline: model 2 IRR 0.78 (95\% Cl $0.61-1.00)$ for beta blocker use alone; 0.81 (95\% Cl 0.64-1.03) for beta blocker in combination with other drugs. None of the other drug categories was significantly associated with cognitive decline.

Pepine 2003 [34] No significant differences in primary outcome (first occurrence of all-cause death, nonfatal Ml or nonfatal stroke) were seen between the calcium antagonist group and the non-calcium antagonist group (RR $0.98 \mathrm{Cl} 0.90-1.06$ ). No significant differences were seen in these outcomes analysed individually.

In the subgroup analysis of patients $>70$ years the primary outcome occurred in 596 of the 3694 patients of the calcium antagonist group (16.3\%) and in 664 of the 3829 patients of the non- calcium antagonist group (17.34\%). RR 0.93 (95\% Cl 0.84-1.03) [p-values missing]

Ruwald 2012 [40] In this post-hoc analysis of the LIFE study, patients were divided in subgroups of aged 66 or younger and aged 67 or older. In the older subgroup, losartan significantly reduced the risk of the composite primary endpoint of cardiovascular death, nonfatal stroke or nonfatal $\mathrm{Ml}$ compared to atenolol (HR 0.79, 95\% Cl 0.69-0.91). In the younger subgroup the effect was not significant (HR 1.03, 95\% Cl 0.82-1.28). Further subdividing suggested a "cut-off age" of 71 years, above which losartan based treatment is better than atenolol based treatment. 
Table 6 Summary of study findings (Continued)

Testa 2014 [46]

Matsuzaki

2011

Main trial [47]

Ogihara 2012 [41]

MRC 1992 [35] Main trial

Older adults taking atenolol showed a greater mortality and higher pulse arterial pressure values than those not taking atenolol (73.9\% vs 55.0\%; $p=0.047$ and $74.7 \pm 14.1$ vs $63.0 \pm 14.2 \mathrm{mmHg}, P<0.001$, respectively). Cox regression analysis showed that atenolol use (HR 1.91; $95 \% \mathrm{Cl} 1.04-4.31 ; p=0.04)$ and pulse arterial pressure (HR 1.02; $95 \% \mathrm{Cl} 1.01-1.03$; $p=0.032$ ) were predictive of long-term mortality.

For the subgroup $\geq 70$ years: There are no statistical significant differences for the primary cardiovascular endpoint in people over 70 years regarding the three intervention arms.

\section{Primary cardiovascular composite endpoint:}

Hazard ratio $(95 \% \mathrm{Cl})$

beta blocker/ARB:

$<70$ years: $1.24(0.72-2.12)$

$\geq 70$ years: $1.21(0.63-2.33)$

Overall population: 1.22 (0.80-1.85)

ARB/TD:

$<70$ years: $1.59(0.83-3.02)$

$\geq 70$ years: $0.97(0.50-1.91)$

Overall population: $1.26(0.80-2.01)$

beta blocker/TD:

$<70$ years: $1.96(1.05-3.66)$

$\geq 70$ years:1.18 $(0.61-2.27)$

Overall population: $1.54(0.98-2.41)$

The number of patients who discontinued the trial because of serious adverse events was $12(1.1 \%), 11(1.0 \%)$, and 11 (1.0\%) in the benidipine-ARB, benidipine-beta blocker, and benidipine-thiazide groups, respectively. The percentage of adverse events was similar among the treatment groups: 505 (45.5\%), 495 (45.5\%), and 522 (47.7\%) patients reported adverse events. The following adverse events occurred more frequently in another group or in two groups: bradycardia (benidipine-beta blocker, $P<0.0001$ ), hyperkalemia (benidipine-ARB, $P=0.0395$ ), vertigo (benidipine-b-blocker and benidipinethiazide, $P=0.0188$.

In this analysis of the COPE trial with 3293 patients in the subgroup of patients aged 65 years or older, benididpe + beta blocker reduced less fatal and non-fatal strokes than benidipine + TD (HR 2.74 (1.08-6-96) and benidipine +beta blocker was associated with more new onset diabetes than benidipine +ARB (HR 2.47 (1.03-5.91). There was no significant difference regarding the composite primary endpoint, cardiovascular endpoints, and all-cause mortality (benidipine plus beta blocker vs. benidipine plus angiotensine receptor blocker HR 0.99 (0.54-1.82); benidipine plus beta blocker vs. benidipine plus thiazide diuretic HR $1.34(0.69-2.60)$

Overall, the beta blocker group had significantly more withdrawals than the diuretic group for both suspected major side effects (beta blocker 30.2\% ( $n=333)$; diuretic 14.8\% ( $n=160)$; placebo 3.7\% $(n=82)$, and inadequate blood pressure control (beta blocker $n=12$; diuretic $n=1$; placebo $n=175$ ). After adjusting for baseline characteristics the diuretic group had significantly reduced risk of stroke (31\% $95 \mathrm{Cl} 3 \%$ to $51 \%, p=0.04$ ), coronary events ( $44 \% 95 \mathrm{Cl} 21 \%$ to $60 \%$, $p=0.0009$ ), and all cardiovascular events (35\% $95 \mathrm{Cl} 17 \%$ to $49 \%, p=0.0005)$ compared with the placebo groups. The beta blocker group showed no significant reductions in these end points.

\section{Outcome, no.} events

Stroke fata

Stroke-nonfatal

Stroke total

Coronary events

fatal

Coronary events-

non-fatal

All cardiovascular

events

All cardiovascular deaths

All deaths

Stroke-nonfatal

Stroke total
Diuretic $(n=1081,6290$ patient years)

\section{6}

$$
29
$$

45

33

15

107

66

134

Beta blocker $(n=1102$ 6330 patient years)

\section{1}

35

56
Placebo $(n=2213$

12,735 patient years)

42

92

134

49

309

180

315

Placebo $(n=2213$ 12,735 patient years)
Relative risk per event/treatment $(95 \% \mathrm{Cl}, p$ value) Based on own calculations:

$0.78(0.44-1.38, p=0.39)$

$0.65(0.43-0.97, p=0.04)$

$0.69(0.49-0.96, p=0.03)$

$0.61(0.42-0.90, p=0.01)$

$0.63(0.35-1.11, p=0.11)$

$0.71(0.58-0.87, p=0.0012)$

$0.75(0.57-0.99, p=0.04)$

$0.87(0.72-1.05, p=0.15)$

$1.00(0.60-1.69, p=0.99)$

$0.76(0.52-1.11, p=0.16)$

$0.84(0.62-1.14, p=0.26)$ 
Table 6 Summary of study findings (Continued)

\begin{tabular}{|c|c|c|c|c|}
\hline & $\begin{array}{l}\text { Coronary events } \\
\text { fatal }\end{array}$ & 52 & 110 & $0.95(0.69-1.31, p=0.75)$ \\
\hline & $\begin{array}{l}\text { Coronary events- } \\
\text { non-fatal }\end{array}$ & 28 & 49 & $1.15(0.73-1.82, p=0.56)$ \\
\hline & $\begin{array}{l}\text { All cardiovascular } \\
\text { events }\end{array}$ & 151 & 309 & $0.98(0.82-1.18, p=0.84)$ \\
\hline & $\begin{array}{l}\text { All cardiovascular } \\
\text { deaths }\end{array}$ & 95 & 180 & $1.06(0.84-1.34, p=0.63)$ \\
\hline & All deaths & 167 & 315 & $1.06(0.90-1.27, p=0.48)$ \\
\hline \multirow{13}{*}{$\begin{array}{l}\text { Bird } 1990[36] \\
\text { Study based on the } \\
\text { MRC trial population }\end{array}$} & $\begin{array}{l}\text { Blood pressure } \\
\text { (mean } \mathrm{mmHg})\end{array}$ & Diuretic group & Beta blocker group & Placebo groups \\
\hline & 1 month & $152 / 80(n=635)$ & $159 / 79(n=624)$ & $166 / 85(n=1303)$ \\
\hline & 9 months & $149 / 79(n=582)$ & $156 / 79(n=481)$ & 167/86 (1156) \\
\hline & \multicolumn{4}{|c|}{ Depression Questionnaire (\% of participants with depressed mood) } \\
\hline & 1 month & $9.1(n=551)$ & $11.0(n=554)$ & $8.8(n=1147)$ \\
\hline & 9 months & $10.4(n=550)$ & $10.4(n=453)$ & $9.2(n=1092)$ \\
\hline & \multicolumn{4}{|c|}{ Paired Associate Learning Test (\% of participants with a score $\leq 15$ ) } \\
\hline & 1 month & $21.0(n=593)$ & $20.0(n=579)$ & $20.0(n=1212)$ \\
\hline & 9 months & $21.2(n=556)$ & $19.9(n=485)$ & $18.5(n=1116)$ \\
\hline & \multicolumn{4}{|c|}{ The Trial-Making Test (seconds) } \\
\hline & $\begin{array}{l}1 \text { month, mean } \\
\text { (SD) }\end{array}$ & $54.3(23.9)(n=593)$ & $54.7(23.9) n=580)$ & $55.5(27.7)(n=1221)$ \\
\hline & $\begin{array}{l}9 \text { months, mean } \\
\text { (SD) }\end{array}$ & $52.4(33.5)(n=560)$ & $53.1(28.2) n=460)$ & $52.2(25.6)(n=1122)$ \\
\hline & \multicolumn{4}{|c|}{$\begin{array}{l}\text { Antihypertensive treatment with either } 25 \text { mg hydrochlorothiazide and } 2.5 \mathrm{mg} \text { amiloride or atenolol } 50 \mathrm{mg} \text { for } 9 \text { months } \\
\text { in a population aged between } 65 \text { and } 74 \text { years old with moderate raised blood pressure did not impair cognitive function, } \\
\text { depression, or behavioural changes which would cause concern in confidants. The study did not find a linear relationship } \\
\text { between blood pressure levels in their moderately elevated range and psychometric test scores, nor showed that lowering } \\
\text { of blood pressure levels is associated with any impairment of performance on these tests }\end{array}$} \\
\hline Carr 2012 [42] & \multicolumn{4}{|c|}{$\begin{array}{l}\text { This secondary analysis of the MRC trial found evidence that atenolol does not perform as well as the hydrochlorothiazide } \\
\text { plus amiloride (Moduretic } \odot \text { ) in terms of stroke prevention: Moduretic } 41.6 \text { number of events } / 1000 \text { patient years, beta } \\
\text { blocker } 50.8 \% \text { and placebo } 60.5 \% \text { (statistical tests missing). For stroke, we found that after adjusting for current systolic } \\
\text { blood pressure variability in systolic blood pressure over time, as measured by the standard residual or root successive } \\
\text { variance, contained significant prognostic capability: } \\
\text { the rate ratio associated with an increase of } 1 \text { standard deviation in the standard residual was } 1.2595 \% \mathrm{Cl} 0.86-1.81 \text { and } \\
1.1695 \% \mathrm{Cl} 0.85-1.59 \text { for the root successive variance. }\end{array}$} \\
\hline Lever $1992[48]$ & \multicolumn{4}{|c|}{$\begin{array}{l}\text { In this article reporting on the MRC trial, the beta blocker group showed no significant differences to placebo regarding } \\
\text { the outcomes stroke, coronary events, all cardiovascular events and all-cause mortality. }\end{array}$} \\
\hline Lever 1993 [39] & \multicolumn{4}{|c|}{$\begin{array}{l}\text { In this article reporting on the MRC trial, a RCT with } 4396 \text { patients (mean age } 70.3 \text { years) randomized to beta blocker, } \\
\text { diuretic or placebo, the beta blocker group showed no significant differences to placebo regarding the outcomes stroke, } \\
\text { coronary events, all cardiovascular events and all-cause mortality. }\end{array}$} \\
\hline
\end{tabular}

Legend: $R R$ relative risk, $C l$ confidence interval, $S D$ standard deviation, $B$ beta blocker, $C$ comparator, NR not reported, IRR incidence risk ratio, $H R$ hazard ratio, $T D$ thiazide diuretic, $A R B$ angiotensin receptor blocker, $A C E$ angiotensin converting enzyme (inhibitor), $C H D$ chronic heart disease, $C H F$ chronic heart failure, $C V D$ cardiovascular disease, $M I$ myocardial infarction, $R C T$ randomized controlled trial, FU Follow Up

[53]. Details of the studies can be seen in Additional file 2 . These references did not meet the inclusion criteria of our systematic review because of the age of the included participants. They were counted as being relevant to recommendations principally because they provided information about the risks and benefits of beta blockers for the management of hypertension in younger populations.

Three of the additional references evaluated atenolol $[50,53,54]$. Atenolol was reported to be associated with an increased risk of stroke compared with other antihypertensive agents [54] and to be less effective at preventing cardiovascular morbidity and death than losartan [53]. However, in a systematic review no statistical differences were found between atenolol and placebo regarding the risk of fatal and non-fatal stroke, major vascular events, death from all causes, and death from cardiovascular causes [50]. The other five additional references evaluated beta blockers $[17,38,49,51,52]$ in general. 


\begin{tabular}{|c|c|c|c|c|c|c|c|c|}
\hline \multirow[b]{2}{*}{ Author year } & \multirow[b]{2}{*}{$\begin{array}{l}\text { Type of } \\
\text { study }\end{array}$} & \multicolumn{2}{|c|}{ Selection bias } & \multirow{2}{*}{$\begin{array}{l}\text { Performance } \\
\text { bias }\end{array}$} & \multirow{2}{*}{$\begin{array}{l}\begin{array}{c}\text { Detection } \\
\text { bias }\end{array} \\
\begin{array}{l}\text { Blinding of } \\
\text { outcome } \\
\text { assessment }\end{array}\end{array}$} & \multirow{2}{*}{\begin{tabular}{l} 
Attrition \\
\multicolumn{1}{c}{ bias } \\
Incomplete \\
outcome \\
data
\end{tabular}} & \multirow{2}{*}{$\begin{array}{c}\begin{array}{c}\text { Reporting } \\
\text { bias }\end{array} \\
\text { Selective } \\
\text { reporting }\end{array}$} & \multirow{2}{*}{$\begin{array}{l}\text { Other bias } \\
\text { (e.g. power } \\
\text { calculation, } \\
\text { ITT } \\
\text { analysis, } \\
\text { contaminati } \\
\text { on bias) }\end{array}$} \\
\hline & & $\begin{array}{l}\text { Random } \\
\text { sequence } \\
\text { generation }\end{array}$ & $\begin{array}{l}\text { Allocation } \\
\text { concealment }\end{array}$ & & & & & \\
\hline Coope 1986 [37] & $\mathrm{RCT}$ & $\overline{\mathrm{LR}}$ & LR & UR & UR & UR & $\overline{\mathrm{LR}}$ & HR \\
\hline Pepine 2003 [34] & RCT & LR & LR & HR & UR & UR & HR & HR \\
\hline Ruwald $2012[40]^{1}$ & $\begin{array}{c}\text { RCT } \\
\text { (post hoc } \\
\text { analysis) }\end{array}$ & LR & LR & LR & LR & LR & LR & HR \\
\hline $\begin{array}{c}\text { COPE main trial } \\
\text { Matsuzaki } 2011 \text { [47], }\end{array}$ & $\mathrm{RCT}$ & LR & LR & HR & LR & UR & UR & HR \\
\hline $\begin{array}{l}\text { Secondary analysis of } \\
\text { the COPE trial: } \\
\text { Ogihara } 2012[41]\end{array}$ & $\mathrm{RCT}$ & LR & LR & HR & LR & LR & UR & HR \\
\hline $\begin{array}{l}\text { MRC main trial } \\
1992[35]\end{array}$ & $\mathrm{RCT}$ & UR & UR & UR & LR & HR & $\mathrm{LR}$ & HR \\
\hline $\begin{array}{l}\text { Secondary analyses of } \\
\text { the MRC trial: } \\
\text { Bird 1990 [36], Carr } \\
\text { 2012 [42], Lever 1992 } \\
\text { [38], Lever 1993 [39] }\end{array}$ & $\mathrm{RCT}$ & UR & UR & UR & LR & HR & LR & HR \\
\hline
\end{tabular}

Fig. 2 Quality appraisal for intervention studies. RCT = randomized controlled trial, $L R=$ low risk of bias, HR = high risk of bias, UR = unclear (insufficient information to permit judgement of low risk or high risk), ITT = intention-to-treat analysis, COPE = Combination Therapy of Hypertension to Prevent Cardiovascular Events, MRC = Medical Research Council. ${ }^{1}$ Risk of bias assessed on study protocol [56]

Although the risk for stroke was lower for beta blockers compared to placebo, there was an increase in stroke in beta blockers compared to calcium channel blockers and renin-angiotensin system inhibitors [17]. This finding is in the same direction as the results reported by Khan et al. [33] for the group of studies enrolling older patients. Total mortality and cardiovascular disease were also higher for beta blockers compared to calcium channel blockers [17]. Furthermore, it was reported that second line beta blockers reduced blood pressure but with little or no effect on pulse pressure compared to thiazides [46]. The Canadian Hypertension Education Program papers were the only sources that recommended beta blockers as first line therapy for patients $<60$ year with coronary artery disease [37].

These additional references provide preliminary evidence that certain risks like fatal and non-fatal stroke, major vascular events, cardiovascular morbidity, death from all causes, and death from cardiovascular causes may be increased with the use of beta blockers compared to other antihypertensive treatments. The uncertainties regarding these risks imply that independently from the age group beta blockers should be prescribed with caution for the treatment of hypertension.

\section{Recommendations}

We developed recommendations following a standardised schema and reflecting the strength and the quality of the evidence. From the results of our systematic review and the additional "references of interest" we were able to develop three recommendations in two meetings by AV (researcher and clinician), YVM (researcher) and ARG (researcher and geriatrician).AS participated in one of these as a senior clinician and researcher. The three recommendations were later discussed and confirmed with IK and MMV for their inclusion in the Comprehensive Medication Review (CMR) tool, which was developed within the PRIMA-eDS project.

The three recommendations we developed are about the discontinuation of beta blocker use in older adults with hypertension (Table 1). The recommendations were considered as strong recommendations. The quality was downgraded to low for indirectness.

\section{Discussion}

This systematic review aimed to explore the effectiveness and safety of beta blockers for the management of hypertension in older adults ( $\geq 65$ years). A further aim was to develop recommendations on when the use of beta blockers could be discontinued or its dose could be reduced in the treatment of hypertension in older adults.

\section{Summary of main results}

One meta-analysis, four randomized controlled trials, six secondary analyses (post hoc analyses) of randomized controlled trials, and four observational studies were included in our systematic review. The included studies were heterogeneous in terms of study designs, interventions, settings, participants, reporting and definitions of 


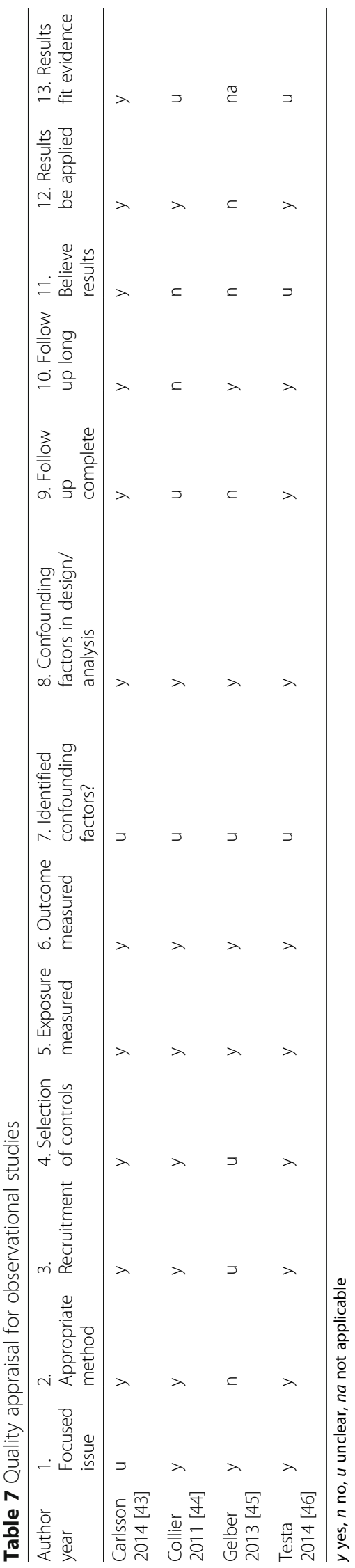


outcome measurements, as well as length of follow-up. The included studies reported on mortality, cardiovascular events including stroke, cognitive impairment or cognitive status and adverse events. We did not find studies analysing the effect on quality of life, life expectancy, hospitalisation, functional impairment or functional status, renal failure, or safety endpoints. The results of the included studies suggest that beta blockers appear to increase the risk of death, stroke and myocardial infarction analysed together as a composite outcome if compared to other antihypertensive agents [31]. Further, beta blockers showed no benefit compared to other antihypertensive agents or placebo regarding the single outcome of mortality. They appear to be less effective than other antihypertensive agents in reducing cardiovascular events. Regarding the endpoint of cognitive impairment/decline, there seems to be a trend towards a decreased risk associated with beta blocker use versus no treatment. Regarding the single endpoint of stroke we found contradictory results.

In addition to the results of the effectiveness of beta blockers identified in the present review, also some issues regarding medication safety have to be risen. First, high cross-over-rates occurred in two studies [32, 34], where a high cross over from the beta blocker group to the comparison group occurred. Pepine et al. [32] gave the following possible reasons for this: physician bias against beta blockers, and suspected higher metabolic complications or previous intolerances against beta blockers.

Second, in another study [33], withdrawal of patients due to suspected major side effects and inadequate blood pressure control in the beta blocker group was significantly higher than in the comparison group.

Regarding the quality of the included studies and the interpretation of the results, a major limitation was that the results are partly based on subgroup analyses (3 out of 5 studies) [31, 32, 34] and secondary analyses of randomized controlled trials [36, 39-42, 48]. Subgroup analyses and secondary analyses in general are used for generating hypotheses and not for testing hypotheses. The included meta-analysis has some major limitations and it can be argued that the systematic review on which it is based does not fulfil the criteria for a systematic review [19]. Only three of the 11 studies included in the subgroup analysis of older people in the meta-analysis were considered eligible for our review. The reporting of the participants' characteristics was very poor with regard to their comorbidities, use of concomitant drugs, frailty, and cognitive status. Thus, the generalizability of the results to the heterogeneous group of older people is limited as the evidence is indirect. However, it could be expected that concerns with regard to safety and benefits of beta blockers for the management of hypertension identified in the present study may be even higher for frailer older people.

\section{Strengths and limitations}

We conducted this systematic review following an adaption of the standard methodology recommended by the Cochrane collaboration [19] and the PRISMA statement [20]. The search strategy comprised a stepwise approach searching first for systematic reviews and meta-analysis (searches 1 and 2), and then for additional relevant controlled intervention and observational studies (searches 3A and 3B). We restricted search 3B to the period 20112016, as earlier literature had already been covered in the process of searches 1, 2 and 3A. Taking into account that the new update of the Cochrane Review of Wiysonge published in 2017 [22] did not identify any additional studies that would be eligible for our review (and the review does not apply any age restrictions), it is very unlikely that any eligible studies have been missed by our search strategy. We excluded a large number of studies because they were either not focussing on beta blockers or they had been carried out in younger populations ( $<65$ years). Our search strategy included specific terms for older adults. This might have limited our ability to capture all studies that include evidence on older adults carrying a risk of missing studies like in a rapid review. But we believe our stepwise approach overcomes some of the limitations of a rapid review, for which there is a lack of published guidelines or explicit methods [51,52]. Unlike our review, some rapid reviews do not have clear research questions including participants, interventions, comparisons, outcomes, and study design (PICOS) [51].

Three of the four individual randomized controlled trials we included $[34,35,37]$ also formed part of the included subgroup analysis of the meta-analysis [33]. Although this does carry a risk of "double-counting", we decided to include both in our systematic review as the overlap was small ( 3 out of 11 trials in the meta-analysis) and the metaanalysis did not examine these studies to the same level of detail as we are doing in our systematic review.

Two of the secondary analyses [39, 48] did not really provide any new data and they also could have been treated as duplicates but as they formally met our inclusion criteria we included them as secondary analyses of the MRC study.

In our systematic review we only found studies specifically addressing Atenolol. It therefore remains unclear whether the results of these studies are also applicable for newer beta blockers such as metoprolol or bisoprolol.

\section{Implications for future research}

Older adults comprise the great majority of consumers of drugs used in the treatment of chronic diseases such as hypertension. Yet, there has been little work to systematically assess the research evidence for usage in this population ( $\geq 65$ years with polypharmacy and/or multimorbidity). Research groups should focus on clinically relevant 
outcomes i.e. mortality, cardiovascular diseases (including stroke) or hospital admissions (drug related) whenever applicable, as these are critical in the context of beta blocker treatment in hypertension. We also want to mention that there are a number of other important endpoints, e.g. quality of life, functional and cognitive status which should be considered especially in studies on older populations. A proper power calculation and definition of a primary endpoint (single or composite) is important to show an effect and to avoid multiple testing. In most studies important information is lacking in the methods section. Study authors should provide a clear and comprehensive description of the population (including comorbidities), study design and intervention. We highly recommend researchers to use the Consolidated Standards of Reporting Trials statement (CONSORT) [55] to improve the quality of reporting of randomized controlled trials.

\section{Conclusions}

This is one of the first systematic reviews exploring the risks and benefits of beta blockers in treating hypertension in aged populations ( $>65$ years) on patient relevant outcomes. This study highlights the limited number of studies that have been carried out on this issue. Furthermore, the quality of current evidence to interpret the benefits of beta blockers in hypertension is generally weak. It cannot be recommended to use beta blockers in older adults as a first line agent for hypertension. Further studies of good quality should analyse the risks and benefits of beta blockers in older people with hypertension taking into account their comorbidities, use of concomitant medications, functional and cognitive status, and evaluating clinically relevant outcomes for this population.

\section{Additional files}

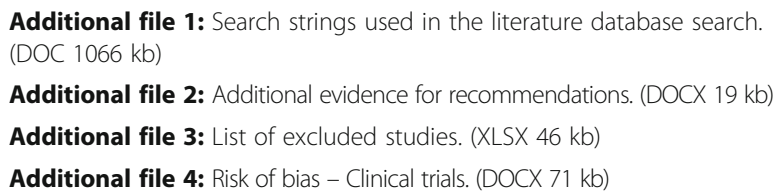

\section{Abbreviations}

ALT: Anne-Lisa Teichmann; AMSTAR: A Measurement Tool to Assess Systematic Reviews; AR: Anja Rieckert; ARG: Anna Renom-Guiteras; AS: Andreas Sönnichsen; AV: Anna Vögele; AW: Adrine Woodham; BF: Barbara Faller; CASP: Critical Appraisal Skills Programme; CMR: Comprehensive Medication Review; DARE: Database of Abstracts or Reviews of Effects; DR: David Reeves; EMBASE: Excerpta Medica dataBASE; GRADE: Grading of Recommendations Assessment, Development and Evaluation; HTA: Health Technology Assessment; IK: Ilkka Kunnamo; IPA: International Pharmaceutical Abstracts; LS: Lisa Schlender; MMV: Minna Marttila Vaara; PICOS: Population, Intervention, Comparison, Outcomes and Study design; PRIMAeDS: Polypharmacy in chronic diseases: Reduction of Inappropriate Medication and Adverse drug events in elderly populations by electronic Decision Support; PRISMA: Preferred Reporting Items for Systematic Reviews and Meta-Analyses; SW: Sabine Weißbach; TJ: Tim Johansson; YM: Yolanda Veronica Martinez

\section{Acknowledgements}

We would like to thank Adrine Woodham for conducting the update databases searches and Christina Sommerauer, Barbara Faller and Sabine Weissbach for selecting studies and conducting data extractions. We also would like to thank Minna Marttila Vaara and Ilkka Kunamo for providing their expertise during the discussion of recommendations.

\section{Funding}

The PRIMA-eDS study was supported by a grant from the European Commission within the 7th Framework Programme (Grant No. 305388-2). The work of YVM was also supported by a grant from the NIHR Greater Manchester Primary Care Patient Safety Translational Research Centre. The publication charge was funded by the University of Witten/Herdecke.

\section{Availability of data and materials \\ The data supporting the conclusions of this article is included within the article (and it's additional files).}

\section{About this supplement}

This article has been published as part of BMC Geriatrics Volume 17 Supplement 1, 2017: The Evidence Base of Frequently prescribed drugs in older Patients: A series of systematic reviews as a basis for recommendations in the PRIMA-eDS-tool to reduce inappropriate polypharmacy. The full contents of the supplement are available online at https://bmcgeriatr.biomedcentral.com/articles/supplements/volume-17-supplement-1.

\section{Authors' contributions}

AS, ARG and YVM conceptualised the study. AV, TJ, YMV, CS, ALT, SW, AR, LS and BF selected the included studies. AV, TJ, YMV, CS, ALT, SW,AR, LS and BF extracted data and conducted quality appraisal. AV, ARG and YVM prepared the recommendations. AV and TJ drafted the manuscript, supported by YVM, $A R G$ and DR. All the authors critically reviewed the drafts and approved the final manuscript.

Ethics approval and consent to participate

Not applicable

Consent for publication

Not applicable

\section{Competing interests}

The authors declare that they have no competing interests.

\section{Publisher's Note}

Springer Nature remains neutral with regard to jurisdictional claims in published maps and institutional affiliations.

\section{Author details}

${ }^{1}$ South Tyrolean Academy of General Practice, via dei Vanga, Bolzano, Italy. ${ }^{2}$ Institute of General Practice and Family Medicine, Paracelsus Medical University, Strubergasse, Salzburg, Austria. ${ }^{3}$ Institute of General Practice and Family Medicine, Faculty of Health, University of Witten/Herdecke, Alfred-Herrhausen-Straße, Witten, Germany. ${ }^{4}$ Department of Geriatrics, University Hospital Parc de Salut Mar, Passeig Marítim, Barcelona, Spain. ${ }^{5} \mathrm{NIHR}$ School for Primary Care Research, Manchester Academic Health Science Centre, Oxford Rd, Manchester, UK.

Published: 16 October 2017

\section{References}

1. Kearney PM, Whelton M, Reynolds K, Muntner P, Whelton PK, He J. Global burden of hypertension: analysis of worldwide data. Lancet. 2005;365(9455):217-23.

2. Organization WH. World Health Statistics. In. Global Health Observatory (GHO) Data; 2015.

3. Mancia G, Fagard R, Narkiewicz K, Redon J, Zanchetti A, Böhm M, Christiaens T, Cifkova R, De Backer G, Dominiczak A, et al. 2013 ESH/ESC guidelines for the management of arterial hypertension: the Task Force for the Management of Arterial Hypertension of the European Society of Hypertension (ESH) and of the European Society of Cardiology (ESC). Eur Heart J. 2013;34(28):2159-219. 
4. Turnbull F, Neal B, Ninomiya T, Algert C, Arima H, Barzi F, Bulpitt C, Chalmers J, Fagard R, Gleason A, et al. Effects of different regimens to lower blood pressure on major cardiovascular events in older and younger adults: meta-analysis of randomised trials. BMJ. 2008;336(7653):1121-3.

5. Staessen JA, Gasowski J, Wang JG, Thijs L, Den Hond E, Boissel JP, Coope J, Ekbom T, Gueyffier F, Liu L, et al. Risks of untreated and treated isolated systolic hypertension in the elderly: meta-analysis of outcome trials. Lancet. 2000;355(9207):865-72

6. Vasan RS, Beiser A, Seshadri S, Larson MG, Kannel WB, D'Agostino RB, Levy D. Residual lifetime risk for developing hypertension in middleaged women and men: The Framingham Heart Study. JAMA. 2002; 287(8):1003-10.

7. Lewington S, Clarke R, Qizilbash N, Peto R, Collins R, Collaboration PS. Agespecific relevance of usual blood pressure to vascular mortality: a metaanalysis of individual data for one million adults in 61 prospective studies. Lancet. 2002;360(9349):1903-13.

8. Logan AG. Hypertension in aging patients. Expert Rev Cardiovasc Ther 2011;9(1):113-20.

9. Chien CC, Yen CS, Wang JJ, Chen HA, Chou MT, Chu CC, Chio CC, Hwang JC, Wang HY, Lu YH, et al. Reverse epidemiology of hypertension-mortality associations in hemodialysis patients: a long-term population-based study. Am J Hypertens. 2012;25(8):900-6.

10. Oates DJ, Berlowitz DR, Glickman ME, Silliman RA, Borzecki AM. Blood pressure and survival in the oldest old. J Am Geriatr Soc. 2007;55(3):383-8.

11. Perry HM, Davis BR, Price TR, Applegate WB, Fields WS, Guralnik JM, Kuller L, Pressel S, Stamler J, Probstfield JL. Effect of treating isolated systolic hypertension on the risk of developing various types and subtypes of stroke: the Systolic Hypertension in the Elderly Program (SHEP). JAMA. 2000; 284(4):465-71.

12. Group JS. Principal results of the Japanese trial to assess optimal systolic blood pressure in elderly hypertensive patients (JATOS). Hypertens Res. 2008;31(12):2115-27.

13. Onder G, Landi F, Fusco D, Corsonello A, Tosato M, Battaglia M, Mastropaolo S, Settanni S, Antocicco M, Lattanzio F. Recommendations to prescribe in complex older adults: results of the CRIteria to assess appropriate Medication use among Elderly complex patients (CRIME) project. Drugs Aging. 2014;31(1):33-45.

14. Capuano A, Irpino A, Gallo M, Ferrante L, Illiano ML, Rinaldi B, Filippelli A, Rossi F. Regional surveillance of emergency-department visits for outpatient adverse drug events. Eur J Clin Pharmacol. 2009;65(7):721-8.

15. Ruiter R, Visser LE, Rodenburg EM, Trifirò G, Ziere G, Stricker BH. Adverse drug reaction-related hospitalizations in persons aged 55 years and over: a population-based study in the Netherlands. Drugs Aging. 2012;29(3):225-32.

16. Moderately elevated blood pressure: a Report from the SBU the Swedish Council on Technology Assessments in Health Care. revision of the SBU Report from 2004. No 170/1U. In.: Swedish Council on Technology Assessment in Health Care; 2008.

17. Wiysonge SC, Bradley HA, Volmink J, Mayosi BM, Mbewu A, Opie LH. Betablockers for hypertension. Cochrane Database Syst Rev. 2012;11

18. Messerli FH. The age factor in hypertension. Hosp Pract (Off Ed). 1986;21(1): 103-5. 109-112

19. Higgins JPT GS. Cochrane Handbook for Systematic Reviews of Intervention Versions 5.1.0 [updated March 2011]. The Cochrane Collaboration; 2011. http://handbook.cochrane.org/. last accessed 20 Jan 2016.

20. Liberati A, Altman DG, Tetzlaff J, Mulrow C, Gøtzsche PC, loannidis JP, Clarke M, Devereaux PJ, Kleijnen J, Moher D. The PRISMA statement for reporting systematic reviews and meta-analyses of studies that evaluate healthcare interventions: explanation and elaboration. BMJ. 2009;339:b2700.

21. Martinez YM, Renom-Guiteras A, Reeves D, Ediriweera de Silva RE, Esmail A, Kunnamo I, Rieckert A, Sommerauer C, Sönnichsen A. A set of systematic reviews to help reduce inappropriate. BMC Geriatrics. 2017;17(1). In press.

22. Wiysonge CS, Bradley HA, Volmink J, Mayosi BM, Opie LH. Beta-blockers for hypertension. Cochrane Database Syst Rev. 2017;1:CD002003.

23. Sönnichsen A, Trampisch US, Rieckert A, Piccoliori G, Vögele A, Flamm M, Johansson T, Esmail A, Reeves D, Löffler C, et al. Polypharmacy in chronic diseases-Reduction of Inappropriate Medication and Adverse drug events in older populations by electronic Decision Support (PRIMA-eDS): study protocol for a randomized controlled trial. Trials. 2016;17:57.

24. Shea BJ, Grimshaw JM, Wells GA, Boers M, Andersson N, Hamel C, Porter AC, Tugwell P, Moher D, Bouter LM. Development of AMSTAR: a measurement tool to assess the methodological quality of systematic reviews. BMC Med Res Methodol. 2007;7:10
25. Shea BJ, Hamel C, Wells GA, Bouter LM, Kristjansson E, Grimshaw J, Henry DA, Boers M. AMSTAR is a reliable and valid measurement tool to assess the methodological quality of systematic reviews. J Clin Epidemiol. 2009;62(10):1013-20.

26. Higgins JPT DG, Jonathan ACS. Chapter 8: Assessing risk of bias in included studies in Cochrane Handbook for Systematic Reviews of Interventions http://handbook.cochrane.org/chapter $8 / 8$ assessing risk of bias in included studies.htm. last accessed 20 Jan 2016. In.

27. Critical Appraisal Skills Programme. 11 questions to help you make sense of case control study. 2013. http://casp-uk.net/casp-tools-checklists. In. last accessed 20/1/2016.

28. Critical Appraisal Skills Programme. 12 questions to help you make sense of cohort study. 2013. http://www.casp-uk.net/wp-content/uploads/2011/11/ CASP-Cohort-Study-Checklist-31.05.13.pdf. In. last accessed 20/1/2016.

29. Sayers $\mathrm{A}$. Tips and tricks in performing a systematic review. $\mathrm{Br} J$ Gen Pract. 2007;57(538):425.

30. Guyatt GH, Oxman AD, Kunz R, Falck-Ytter $Y$, Vist GE, Liberati A, Schünemann HJ, Group GW. Going from evidence to recommendations. BMJ. 2008:336(7652):1049-51.

31. Guyatt GH, Oxman AD, Vist GE, Kunz R, Falck-Ytter Y, Alonso-Coello P, Schünemann HJ, Group GW. GRADE: an emerging consensus on rating quality of evidence and strength of recommendations. BMJ. 2008;336(7650):924-6.

32. Guyatt $G H$, Oxman AD, Kunz R, Vist GE, Falck-Ytter $Y$, Schünemann HJ, Group GW. What is \&quot;quality of evidence\&quot; and why is it important to clinicians? BMJ. 2008;336(7651):995-8.

33. Khan N, McAlister FA. Re-examining the efficacy of beta-blockers for the treatment of hypertension: A meta-analysis. Can Med Assoc J. 2006;174(12): 1737-42.

34. Pepine CJ, Handberg EM, Cooper-DeHoff RM, Marks RG, Kowey P, Messerli FH, Mancia G, Cangiano JL, Garcia-Barreto D, Keltai M, et al. A calcium antagonist vs a non-calcium antagonist hypertension treatment strategy for patients with coronary artery disease. The International Verapamil-Trandolapril Study (INVEST): a randomized controlled trial. JAMA. 2003;290(21):2805-16.

35. Medical Research Council trial of treatment of hypertension in older adults: principal results. MRC Working Party. BMJ 1992;304(6824):405-412.

36. Bird AS, Blizard RA, Mann AH. Treating hypertension in the older person: an evaluation of the association of blood pressure level and its reduction with cognitive performance. J Hypertens. 1990:8(2):147-52.

37. Coope J, Warrender TS. Randomised trial of treatment of hypertension in elderly patients in primary care. Br Med J (Clin Res Ed). 1986;293(6555):1145-51.

38. Duodecim Medical Publications Ltd. EBM Guidelines [Internet]: ebm00759 Cerebral Infarction (ischaemic stroke). Helsinki [updated 2013 May17; cited 2016 June 15]. Available from http://www.ebm-guidelines.com. In

39. Lever AF, Brennan PJ. MRC trial of treatment in elderly hypertensives. Clin Exp Hypertens (New York). 1993;15(6):941-52.

40. Ruwald AC, Westergaard B, Sehestedt T, Kjeldsen SE, Lindholm LH, Wachtell $K$, Devereux RB, Ibsen $H$, Nieminen MS, Dahlof B, et al. Losartan versus atenolol-based antihypertensive treatment reduces cardiovascular events especially well in elderly patients: the Losartan Intervention For Endpoint reduction in hypertension (LIFE) study. J Hypertens. 2012;30(6):1252-9.

41. Ogihara T, Matsuzaki M, Umemoto S, Rakugi H, Matsuoka H, Shimada K, Higaki J, Ito S, Kamiya A, Suzuki H, et al. Combination therapy for hypertension in the elderly: a sub-analysis of the Combination Therapy of Hypertension to Prevent Cardiovascular Events (COPE) Trial. Hypertens Res. 2012;35(4):441-8.

42. Carr MJ, Bao Y, Pan J, Cruickshank K, McNamee R. The predictive ability of blood pressure in elderly trial patients. J Hypertens. 2012;30(9):1725-33.

43. Carlsson AC, Wandell P, Sundquist K, Johansson SE, Sundquist J. Effects of prescribed antihypertensives and other cardiovascular drugs on mortality in patients with atrial fibrillation and hypertension: a cohort study from Sweden. Hypertens Res Clin Exp. 2014;37(6):553-9.

44. Collier DJ, Poulter NR, Dahlof B, Sever PS, Wedel H, Buch J, Caulfield MJ, Investigators A. Impact of amlodipine-based therapy among older and younger patients in the Anglo-Scandinavian Cardiac Outcomes Trial-Blood Pressure Lowering Arm (ASCOT-BPLA). J Hypertens. 2011;29(3):583-91.

45. Gelber RP, Ross GW, Petrovitch $H$, Masaki KH, Launer $\sqcup$, White LR. Antihypertensive medication use and risk of cognitive impairment: the Honolulu-Asia Aging Study. Neurology. 2013;81(10):888-95.

46. Testa G, Cacciatore F, Della-Morte D, Mazzella F, Mastrobuoni C, Galizia G, Gargiulo G, Rengo F, Bonaduce D, Abete P. Atenolol use is associated with long-term mortality in community-dwelling older adults with hypertension. Geriatr Gerontol Int. 2014;14(1):153-8. 
47. Matsuzaki M, Ogihara T, Umemoto S, Rakugi H, Matsuoka H, Shimada K, Abe K, Suzuki N, Eto T, Higaki J, et al. Prevention of cardiovascular events with calcium channel blocker-based combination therapies in patients with hypertension: a randomized controlled trial. J Hypertens. 2011;29(8):1649-59.

48. Lever AF. BPJ: MRC trial of treatment in elderly hypertensives. High Blood Press. 1992;:1:132-7.

49. Chen MHJ, Heran BS, Perez MI, Wright JM. Blood pressure lowering efficacy of beta-blockers as second-line therapy for primary hypertension. Cochrane Database Syst Rev. 2010;1

50. De Lima GL, Soares GOB, Saconato H, Atallah AN, da Silva MKE. Beta-blockers for preventing stroke recurrence. Cochrane Database Syst Rev. 2013;5

51. Hackam DG, Khan NA, Hemmelgarn BR, Rabkin SW, Touyz RM, Campbell NR, Padwal R, Campbell TS, Lindsay MP, Hill MD, et al. The 2010 Canadian Hypertension Education Program recommendations for the management of hypertension: part 2 - therapy. Can J Cardiol. 2010;26(5):249-58.

52. Leung AA, Nerenberg K, Daskalopoulou SS, McBrien K, Zarnke KB, Dasgupta K, Cloutier L, Gelfer M, Lamarre-Cliche M, Milot A, et al. Hypertension Canada's 2016 Canadian Hypertension Education Program Guidelines for Blood Pressure Measurement, Diagnosis, Assessment of Risk, Prevention, and Treatment of Hypertension. Can J Cardiol. 2016;32(5):569-88.

53. Dahlof B, Devereux RB, Kjeldsen SE, Julius S, Beevers G, De Faire U, Fyhrquist F, Ibsen H, Kristiansson K, Lederballe-Pedersen O, et al. Cardiovascular morbidity and mortality in the Losartan Intervention For Endpoint reduction in hypertension study (LIFE): A randomised trial against atenolol. Lancet. 2002;359(9311):995-1003.

54. Kuyper LM, Khan NA. Atenolol vs nonatenolol beta-blockers for the treatment of hypertension: a meta-analysis. Can J Cardiol. 2014:30:S47-53.

55. Moher D, Hopewell S, Schulz KF, Montori V, Gøtzsche PC, Devereaux PJ, Elbourne D, Egger M, Altman DG. CONSORT 2010 explanation and elaboration: updated guidelines for reporting parallel group randomised trials. BMJ. 2010;340:8869.

56. Dahlöf B, Devereux R, de Faire U, Fyhrquist F, Hedner T, Ibsen H, Julius S, Kjeldsen $\mathrm{S}$, Kristianson K, Lederballe-Pedersen O, et al. The Losartan Intervention For Endpoint reduction (LIFE) in Hypertension study: rationale, design, and methods. The LIFE Study Group. Am J Hypertens. 1997;10(7 Pt 1):705-13.

\section{Submit your next manuscript to BioMed Central and we will help you at every step:}

- We accept pre-submission inquiries

- Our selector tool helps you to find the most relevant journal

- We provide round the clock customer support

- Convenient online submission

- Thorough peer review

- Inclusion in PubMed and all major indexing services

- Maximum visibility for your research

Submit your manuscript at www.biomedcentral.com/submit 